\title{
The Gaia-ESO Survey: CNO abundances in the open clusters Trumpler 20, NGC 4815, and NGC 6705*
}

\author{
G. Tautvaišiené ${ }^{1}$, A. Drazdauskas ${ }^{1}, \check{S}_{\text {. Mikolaitis }}^{1,2}$, G. Barisevičius $^{1}$, E. Puzeras ${ }^{1}$, E. Stonkuté ${ }^{1}$, Y. Chorniy $^{1}$,

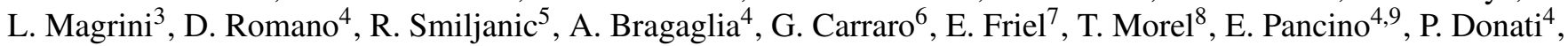 \\ F. Jiménez-Esteban ${ }^{10}$, G. Gilmore ${ }^{11}$, S. Randich ${ }^{3}$, R. D. Jeffries ${ }^{12}$, A. Vallenari ${ }^{13}$, T. Bensby ${ }^{14}$, E. Flaccomio ${ }^{15}$, \\ A. Recio-Blanco ${ }^{2}$, M. T. Costado ${ }^{16}$, V. Hill ${ }^{2}$, P. Jofré ${ }^{11}$, C. Lardo ${ }^{17}$, P. de Laverny ${ }^{2}$, T. Masseron ${ }^{11}$, L. Moribelli ${ }^{3}$, \\ S. G. Sousa ${ }^{18}$, and S. Zaggia ${ }^{4}$ \\ (Affiliations can be found after the references)
}

Received 15 September 2014 / Accepted 20 October 2014

\begin{abstract}
Context. The Gaia-ESO Public Spectroscopic Survey will observe a large sample of clusters and cluster stars, covering a wide age-distancemetallicity-position-density parameter space.

Aims. We aim to determine C, N, and O abundances in stars of Galactic open clusters of the Gaia-ESO survey and to compare the observed abundances with those predicted by current stellar and Galactic evolution models. In this pilot paper, we investigate the first three intermediate-age open clusters.

Methods. High-resolution spectra, observed with the FLAMES-UVES spectrograph on the ESO VLT, were analysed using a differential model atmosphere method. Abundances of carbon were derived using the $\mathrm{C}_{2}$ band heads at 5135 and $5635.5 \AA$. The wavelength interval $6470-6490 \AA$, with CN features, was analysed to determine nitrogen abundances. Oxygen abundances were determined from the [O I] line at $6300 \AA$.

Results. The mean values of the elemental abundances in Trumpler 20 as determined from 42 stars are: $[\mathrm{Fe} / \mathrm{H}]=0.10 \pm 0.08(\mathrm{~s} . \mathrm{d}$.), $[\mathrm{C} / \mathrm{H}]=$ $-0.10 \pm 0.07,[\mathrm{~N} / \mathrm{H}]=0.50 \pm 0.07$, and consequently $\mathrm{C} / \mathrm{N}=0.98 \pm 0.12$. We measure from five giants in $\mathrm{NGC} 4815:[\mathrm{Fe} / \mathrm{H}]=-0.01 \pm 0.04$, $[\mathrm{C} / \mathrm{H}]=-0.17 \pm 0.08,[\mathrm{~N} / \mathrm{H}]=0.53 \pm 0.07,[\mathrm{O} / \mathrm{H}]=0.12 \pm 0.09$, and $\mathrm{C} / \mathrm{N}=0.79 \pm 0.08$. We obtain from 27 giants in $\mathrm{NGC} 6705:[\mathrm{Fe} / \mathrm{H}]=$ $0.0 \pm 0.05,[\mathrm{C} / \mathrm{H}]=-0.08 \pm 0.06,[\mathrm{~N} / \mathrm{H}]=0.61 \pm 0.07,[\mathrm{O} / \mathrm{H}]=0.13 \pm 0.05$, and $\mathrm{C} / \mathrm{N}=0.83 \pm 0.19$. The $\mathrm{C} / \mathrm{N}$ ratios of stars in the investigated open clusters were compared with the ratios predicted by stellar evolutionary models. For the corresponding stellar turn-off masses from 1.9 to $3.3 M_{\odot}$, the observed $\mathrm{C} / \mathrm{N}$ ratio values are very close to the predictions of standard first dredge-up models as well as to models of thermohaline extra-mixing. They are not decreased as much as predicted by the recent model in which the thermohaline- and rotation-induced extra-mixing act together. The average $[\mathrm{O} / \mathrm{H}]$ abundance ratios of NGC 4815 and NGC 6705 are compared with the predictions of two Galactic chemical evolution models. The data are consistent with the evolution at the solar radius within the errors.

Conclusions. The first results of CNO determinations in open clusters show the potential of the Gaia-ESO Survey to judge stellar and Galactic chemical evolution models and the validity of their physical assumptions through a homogeneous and detailed spectral analysis.
\end{abstract}

Key words. stars: abundances - stars: evolution - Galaxy: evolution - open clusters and associations: individual: Trumpler 20 open clusters and associations: individual: NGC 4815 - open clusters and associations: individual: NGC 6705

\section{Introduction}

Carbon, nitrogen, and oxygen $(\mathrm{CNO})$ are important in Galactic and stellar evolution for many reasons. They comprise most of the mass of elements heavier than helium, so their abundances reflect the bulk of chemical enrichment. The CNO elements are among the first elements to form in the nucleosynthesis chain. These elements play important roles in stellar interiors as sources of opacity and energy production through the $\mathrm{CNO}$ cycle, and thus affect the star's lifetime, its position in the Hertzsprung-Russell (HR) diagram, and its heavy-element yields. The CNO isotopes originate in different stages of the evolution of stars of different masses. In the Galaxy, their relative abundances vary spatially and with time. Therefore, they can provide important information about the Galactic chemical evolution. Investigating abundances of $\mathrm{CNO}$ in objects for

* Based on data products from observations made with ESO Telescopes at the La Silla Paranal Observatory under programme ID 188.B-3002 (The Gaia-ESO Public Spectroscopic Survey, PIs G. Gilmore and S. Randich). which the spatial position and age can be determined with the best precision can provide the most valuable information. When studying the evolution of the Galactic disc, such objects, without doubt, are open star clusters (cf. Janes 1979; Panagia \& Tosi 1980; Cameron 1985; Friel 1995; Twarog et al. 1997; Carraro et al. 1998, 2007; Friel et al. 2002; Chen et al. 2003; Salaris et al. 2004; Bragaglia et al. 2008; Sestito et al. 2008; Jacobson et al. 2009; Pancino et al. 2010; Magrini et al. 2009, 2010, 2014; Lépine et al. 2011; Yong et al. 2012, and references therein).

Open star clusters are even more important in giving us the opportunity to investigate stellar evolution. In open clusters we can analyse a number of stars of essentially the same age, distance, and origin, as open cluster stars are most likely formed in the same protocloud of gas and dust (see, e.g., Lada \& Lada 2003; Pallavicini 2003). There is a wide discussion nowadays to assess if open clusters present singe or multiple populations, however, this question is applicable predominantly to massive open clusters (see Cantat-Gaudin et al. 2014, and references therein). If $\mathrm{CNO}$ abundances in cluster members initially were identical, their abundance changes in stellar atmospheres 
of evolved stars are mainly related to internal processes of stellar evolution. This circumstance was exploited in a number of studies of open clusters (Gilroy 1989; Gilroy \& Brown 1991; Luck 1994; Gonzalez \& Wallerstein 2000; Tautvaišienė et al. 2000, 2005; Origlia et al. 2006; Smiljanic et al. 2009; Mikolaitis et al. 2010, 2011a,b, 2012, among others). The observational data have provided evidence not only of the first dredge-up (1DUP; Iben 1965, 1967; Dearborn et al. 1976), which brings the CN-processed material up to the surfaces of low-mass stars when they reach the bottom of the red giant branch (RGB), but also show evidence of extra-mixing, which happens later on the giant branch.

The extra-mixing processes become efficient on the RGB when these stars reach the so-called RGB bump (Charbonnel 1994; Charbonnel et al. 1998). It also has been recognised that alterations of surface abundances depend on stellar evolutionary stage, mass, and metallicity (e.g. Boothroyd \& Sackmann 1999; Gratton et al. 2000; Chanamé et al. 2005; Charbonnel 2006; Eggleton et al. 2006; Cantiello \& Langer 2010; Charbonnel \& Lagarde 2010; Tautvaišienè et al. 2010, 2013; Lagarde et al. 2012).

The nature of the extra mixing itself is still a matter of debate. Currently, thermohaline mixing seems to be the preferred mechanism, as it fulfills most of the requirements to explain the observations (see e.g. Charbonnel \& Zahn 2007; Angelou et al. 2012). Nevertheless, there are still open questions regarding the physical properties of this mechanism itself (Church et al. 2014), its efficiency to transport chemicals (see e.g. Denissenkov \& Merryfield 2011), and whether or not it might be suppressed in the presence of other mixing mechanisms (Maeder et al. 2013). We also note that other extra mixing mechanisms like magnetic buoyancy have been suggested and need verification (Busso et al. 2007; Palmerini et al. 2011a,b). A better characterisation of these processes needs a comprehensive and statistically significant observational investigation in stars of different masses and metallicities.

The Gaia-ESO Spectroscopic Survey (GES, see Gilmore et al. 2012; Randich \& Gilmore 2013) provides an opportunity to address these issues. The large, public spectroscopic survey of the Galaxy using the high-resolution, multi-object spectrograph on the Very Large Telescope (ESO, Chile) is targeting about $10^{5}$ stars in the bulge, thick and thin discs, and halo components, and a sample of up to 80 open clusters of various ages, metallicities, locations, and masses.

In this work, we present investigations of $\mathrm{CNO}$ abundances in the open clusters Trumpler 20 ( $\operatorname{Tr} 20)$, NGC 4815, and NGC 6705 (M 11), which were observed during the first six months of the GES survey. All three clusters are located in the inner part of the Galaxy: Trumpler 20 is at $l=301.475$ and $b=2.221$, NGC 4815 is at $l=303.6$ and $b=-2.1$, and NGC 6705 is at $l=27.307$ and $b=-2.776$.

Trumpler 20 is a relatively old open cluster. Based on the first internal GES data release (GESviDR1Final) and photometric observations, comparisons with three different sets of isochrones yielded consistent determinations of the cluster age of 1.35 to $1.66 \mathrm{Gyr}$, a turn-off mass of $1.9 \pm 0.1 M_{\odot}$, a distance of $3.4-3.5 \mathrm{kpc}$, the Galactocentric radius $\left(R_{\mathrm{GC}}\right)$ of $6.88 \mathrm{kpc}$, and the average iron abundance of 13 members was $[\mathrm{Fe} / \mathrm{H}]=0.17$ (Donati et al. 2014).

NGC 4815 is an intermediate age cluster. It has not been observed spectroscopically before GES. According to GESviDR1Final, a mean $[\mathrm{Fe} / \mathrm{H}]$ was determined to be equal to $+0.03 \pm 0.05$ dex. Comparisons with three different sets of isochrones yielded consistent determinations of the cluster age of 0.5 to $0.63 \mathrm{Gyr}$, a turn-off mass of $2.6 \pm 0.1 M_{\odot}$, a distance of $2.5-2.7 \mathrm{kpc}$, and $R_{\mathrm{GC}}=6.9 \mathrm{kpc}$ (Friel et al. 2014).

NGC 6705 is the youngest among the open clusters in this paper. As determined by Cantat-Gaudin et al. (2014) on the basis of GESviDR1Final, the age of NGC 6705 is in the range from 0.25 to $0.32 \mathrm{Gyr}$, the turn-off mass from 3.47 to $3.2 M_{\odot}$, depending on the adopted stellar model, and the Galactocentric radius equal to $6.3 \mathrm{kpc}$. The average iron abundance of 21 members was $[\mathrm{Fe} / \mathrm{H}]=0.10 \pm 0.06$.

This pilot paper on CNO abundances in the first three intermediate-age open clusters is based on the second internal GES data release (GESviDR2Final).

\section{Observations and method of analysis}

\subsection{Observations}

Observations were conducted with the FLAMES (Fiber Large Array Multi-Element Spectrograph) multi-fiber facility (Pasquini et al. 2002) in spring of 2012 and 2013. Spectra of high-resolving power $(R \approx 47000)$ were obtained with UVES (Ultraviolet and Visual Echelle Spectrograph, Dekker et al. 2000). The spectra were exposed onto two CCDs, resulting in a wavelength coverage of 4700-6840 A with a gap of about $50 \AA$ in the centre. The spectra were reduced with the ESO UVES pipeline and dedicated scripts described by Sacco et al. (2014). Radial velocities (RV) and rotation velocities $(v \sin i)$ were also determined by cross-correlating all the spectra with a sample of synthetic templates specifically derived for the Gaia-ESO project.

The information on radial velocities was particularly useful in determining true members of the stellar clusters. The typical error on RVs is about $0.4 \mathrm{~km} \mathrm{~s}^{-1}$. In this work, we investigate cluster stars identified by Donati et al. (2014) for Trumpler 20, by Friel et al. (2014) for NGC 4815, and by Cantat-Gaudin et al. (2014) for NGC 6705. In cases where additional cluster stars in the Gaia-ESO Survey were observed later, the identical mean radial velocity and dispersion values determined in these studies $\left(-40.26 \pm 0.11 \mathrm{~km} \mathrm{~s}^{-1}\right.$ for Trumpler 20 , and $34.1 \pm 1.5 \mathrm{~km} \mathrm{~s}^{-1}$ for NGC 6705) as well as information of proper motions guided us in identifying the cluster members. A list of the investigated 42 stars in Trumpler 20, 5 stars in NGC 4815, and 27 stars in NGC 6705 as well as some of their parameters are presented in Tables 1-3, respectively. Figures 1-3 show the investigated stars in the colour-magnitude diagrams.

The signal-to-noise ratio $(\mathrm{S} / \mathrm{N})$ in the spectra of the observed cluster stars varies depending on their brightness. The highest $\mathrm{S} / \mathrm{N}$, ranging from 80 to 190 , was achieved for stars in NGC 6705 , from 45 to 65 in NGC 4815 , and from 20 to 70 in Trumpler 20. Examples of stellar spectra with typical $\mathrm{S} / \mathrm{N}$ for stars in each of the clusters are presented in Fig. 4. All the spectra were taken from the GES database ${ }^{1}$. Additional efforts were applied in improving their continuum normalisation. For this purpose we used the SPLAT-VO code ${ }^{2}$.

In the standard procedure of the 1st and 2nd GES data releases, only a single, final file for each star was used for analysis. This file is a sum of all spectra of a particular star taken in

\footnotetext{
1 The operational database has been developed by the Cambridge Astronomical Survey Unit (CASU) based at the Institute of Astronomy at the University of Cambridge. See the website http://casu.ast. cam.ac.uk/gaiaeso/for more information.

2 http://star-wWW.dur.ac.uk/ pdraper/splat/splat-vo/ splat-vo.html
} 
Table 1. Stellar parameters for target stars in Trumpler 20.

\begin{tabular}{|c|c|c|c|c|c|c|c|c|c|}
\hline ID & GES ID & $\begin{array}{c}\text { RA } \\
\operatorname{deg}(\mathrm{J} 2000)\end{array}$ & $\begin{array}{c}\text { Dec } \\
\operatorname{deg}(\mathrm{J} 2000)\end{array}$ & $\begin{array}{c}V \\
\mathrm{mag}\end{array}$ & $\begin{array}{c}B-V \\
\text { mag }\end{array}$ & $\begin{array}{c}\mathrm{RV} \\
\mathrm{km} \mathrm{s}^{-1}\end{array}$ & $S / N$ & $\begin{array}{l}v \sin i^{a} \\
\mathrm{~km} \mathrm{~s}^{-1}\end{array}$ & $\begin{array}{l}v \sin i^{b} \\
\mathrm{~km} \mathrm{~s}^{-1}\end{array}$ \\
\hline 2730 & $12383595-6045245$ & 189.6498 & -60.7568 & 14.942 & 1.376 & -41.39 & 33 & 2.00 & 3.0 \\
\hline 2690 & $12383659-6045300$ & 189.6525 & -60.7583 & 14.572 & 1.442 & -42.57 & 20 & 2.46 & 3.5 \\
\hline 63 & $12385807-6030286$ & 189.7420 & -60.5079 & 13.603 & 1.588 & -42.35 & 71 & 2.61 & 3.5 \\
\hline 292 & $12390411-6034001$ & 189.7671 & -60.5667 & 13.506 & 1.583 & -40.45 & 72 & 2.64 & 2.6 \\
\hline 1082 & $12390478-6041475$ & 189.7699 & -60.6965 & 14.800 & 1.392 & -41.78 & 36 & 2.00 & 3.0 \\
\hline 724 & $12390710-6038057$ & 189.7796 & -60.6349 & 15.018 & 1.346 & -41.44 & 31 & 2.00 & 2.5 \\
\hline 794 & $12391004-6038402$ & 189.7918 & -60.6445 & 13.703 & 1.618 & -41.89 & 57 & 3.30 & 4.0 \\
\hline 582 & $12391114-6036527$ & 189.7964 & -60.6146 & 14.923 & 1.358 & -42.66 & 36 & 2.00 & 3.0 \\
\hline 542 & $12391201-6036322$ & 189.8000 & -60.6089 & 14.690 & 1.332 & -41.80 & 40 & 2.00 & 2.5 \\
\hline 340 & $12391577-6034406$ & 189.8157 & -60.5779 & 14.669 & 1.331 & -40.01 & 36 & 2.00 & 3.5 \\
\hline 770 & $12392585-6038279$ & 189.8577 & -60.6411 & 14.928 & 1.342 & -42.60 & 23 & 2.00 & 3.5 \\
\hline 950 & $12392637-6040217$ & 189.8599 & -60.6727 & 14.831 & 1.384 & -41.85 & 27 & 2.36 & 3.5 \\
\hline 505 & $12392700-6036053$ & 189.8625 & -60.6015 & 14.519 & 1.394 & -40.02 & 46 & 2.00 & 3.5 \\
\hline 894 & $12393132-6039422$ & 189.8805 & -60.6617 & 14.766 & 1.339 & -36.09 & 40 & 2.25 & 3.5 \\
\hline 203 & $12393741-6032568$ & 189.9059 & -60.5491 & 14.865 & 1.338 & -41.44 & 29 & 2.00 & 3.0 \\
\hline 835 & $12393782-6039051$ & 189.9076 & -60.6514 & 14.577 & 1.380 & -40.69 & 32 & 2.00 & 4.5 \\
\hline 1010 & $12394051-6041006$ & 189.9188 & -60.6835 & 14.643 & 1.395 & -42.79 & 32 & 2.26 & 3.5 \\
\hline 923 & $12394122-6040040$ & 189.9218 & -60.6678 & 14.872 & 1.384 & -39.32 & 37 & 2.00 & 3.5 \\
\hline 858 & $12394309-6039193$ & 189.9295 & -60.6554 & 14.676 & 1.433 & -41.82 & 31 & 2.00 & 3.5 \\
\hline 227 & $12394387-6033166$ & 189.9328 & -60.5546 & 14.590 & 1.338 & -41.00 & 33 & 2.00 & 5.0 \\
\hline 346 & $12394419-6034412$ & 189.9341 & -60.5781 & 14.704 & 1.366 & -41.08 & 35 & 2.00 & 3.5 \\
\hline 781 & $12394475-6038339$ & 189.9365 & -60.6428 & 14.610 & 1.395 & -39.37 & 36 & 2.00 & 3.5 \\
\hline 768 & $12394517-6038257$ & 189.9382 & -60.6405 & 14.789 & 1.380 & -41.50 & 38 & 2.00 & 3.5 \\
\hline 791 & $12394596-6038389$ & 189.9415 & -60.6441 & 14.535 & 1.378 & -39.72 & 44 & 2.00 & 3.0 \\
\hline 287 & $12394690-6033540$ & 189.9454 & -60.5650 & 14.781 & 1.346 & -40.99 & 37 & 2.00 & 3.5 \\
\hline 1008 & $12394715-6040584$ & 189.9465 & -60.6829 & 13.949 & 1.606 & -40.30 & 54 & 2.87 & 3.5 \\
\hline 795 & $12394742-6038411$ & 189.9476 & -60.6448 & 14.712 & 1.358 & -40.00 & 32 & 2.00 & 4.0 \\
\hline 246 & $12394899-6033282$ & 189.9541 & -60.5578 & 14.575 & 1.350 & -39.67 & 30 & 2.33 & 4.0 \\
\hline 787 & $12395426-6038369$ & 189.9761 & -60.6436 & 14.597 & 1.385 & -42.45 & 45 & 2.00 & 4.0 \\
\hline 638 & $12395555-6037268$ & 189.9815 & -60.6241 & 14.613 & 1.381 & -41.06 & 57 & 2.00 & 3.0 \\
\hline 430 & $12395569-6035233$ & 189.9820 & -60.5898 & 15.322 & 1.024 & -38.99 & 25 & 4.25 & 8.0 \\
\hline 827 & $12395655-6039011$ & 189.9856 & -60.6503 & 14.805 & 1.384 & -38.99 & 39 & 2.00 & 3.0 \\
\hline 885 & $12395712-6039335$ & 189.9880 & -60.6593 & 14.660 & 1.381 & -42.06 & 42 & 2.00 & 3.0 \\
\hline 399 & $12395975-6035072$ & 189.9990 & -60.5853 & 14.618 & 1.425 & -42.10 & 53 & 2.00 & 4.0 \\
\hline 129 & $12400111-6031395$ & 190.0046 & -60.5276 & 14.716 & 1.420 & -40.90 & 39 & 2.00 & 3.0 \\
\hline 429 & $12400116-6035218$ & 190.0048 & -60.5894 & 14.615 & 1.393 & -40.87 & 43 & 2.00 & 4.0 \\
\hline 911 & $12400260-6039545$ & 190.0108 & -60.6651 & 13.780 & 1.621 & -41.29 & 72 & 2.82 & 4.0 \\
\hline 1044 & $12400278-6041192$ & 190.0116 & -60.6887 & 14.985 & 1.385 & -39.09 & 27 & 2.00 & 3.0 \\
\hline 591 & $12400451-6036566$ & 190.0188 & -60.6157 & 13.690 & 1.668 & -41.65 & 62 & 3.49 & 4.0 \\
\hline 468 & $12400755-6035445$ & 190.0315 & -60.5957 & 13.548 & 1.634 & -41.12 & 60 & 3.51 & 4.5 \\
\hline 679 & $12402229-6037419$ & 190.0929 & -60.6283 & 14.743 & 1.417 & -41.55 & 31 & 2.30 & 5.0 \\
\hline 3470 & $12402480-6043101$ & 190.1033 & -60.7195 & 13.922 & 1.599 & -40.70 & 47 & 3.08 & 4.0 \\
\hline
\end{tabular}

Notes. ID and photometric data were taken from Carraro et al. (2010); ${ }^{(a)}$ values from the GES database; ${ }^{(b)}$ determined in this work.

Table 2. Stellar parameters for target stars in NGC 4815.

\begin{tabular}{rccccccccc}
\hline \hline ID & GES ID & $\begin{array}{c}\text { RA } \\
\operatorname{deg}(\mathrm{J} 2000)\end{array}$ & $\begin{array}{c}\text { Dec } \\
\operatorname{deg}(\mathrm{J} 2000)\end{array}$ & $\begin{array}{c}V \\
\mathrm{mag}\end{array}$ & $\begin{array}{c}V-I \\
\mathrm{mag}\end{array}$ & $\begin{array}{c}\mathrm{RV} \\
\mathrm{km} \mathrm{s}^{-1}\end{array}$ & $S / N$ & $\begin{array}{c}v \sin i^{a} \\
\mathrm{~km} \mathrm{~s}^{-1}\end{array}$ & $\begin{array}{c}v \sin i^{b} \\
\mathrm{~km} \mathrm{~s}^{-1}\end{array}$ \\
\hline 1795 & $12572442-6455173$ & 194.3518 & -64.9215 & 13.482 & 2.273 & -30.11 & 65 & 4.56 & 5.0 \\
358 & $12574328-6457386$ & 194.4303 & -64.9607 & 13.703 & 1.946 & -31.12 & 63 & 4.09 & 5.5 \\
210 & $12575511-6458483$ & 194.4796 & -64.9801 & 14.043 & 1.841 & -29.92 & 53 & 3.25 & 4.5 \\
95 & $12575529-6456536$ & 194.4804 & -64.9482 & 14.128 & 1.730 & -30.75 & 49 & 2.00 & 3.5 \\
106 & $12580262-6456492$ & 194.5109 & -64.9470 & 14.158 & 1.896 & -30.60 & 46 & 3.30 & 6.0 \\
\hline
\end{tabular}

Notes. $V$ and $V-I$ were taken from Prisinzano et al. $(2001) ;{ }^{(a)}$ values from the GES database; ${ }^{(b)}$ determined in this work. 
Table 3. Stellar parameters for target stars in NGC 6705.

\begin{tabular}{|c|c|c|c|c|c|c|c|c|c|}
\hline ID & GES ID & $\begin{array}{c}\text { RA } \\
\operatorname{deg}(\mathrm{J} 2000)\end{array}$ & $\begin{array}{c}\text { Dec } \\
\operatorname{deg}(\mathrm{J} 2000)\end{array}$ & $\begin{array}{c}V \\
\text { mag }\end{array}$ & $\begin{array}{c}B-V \\
\text { mag }\end{array}$ & $\begin{array}{c}\mathrm{RV} \\
\mathrm{km} \mathrm{s}^{-1}\end{array}$ & $S / N$ & $\begin{array}{l}v \sin i^{a} \\
\mathrm{~km} \mathrm{~s}^{-1}\end{array}$ & $\begin{array}{l}v \sin i^{b} \\
\mathrm{~km} \mathrm{~s}^{-1}\end{array}$ \\
\hline 2000 & $18502831-0615122$ & 282.6180 & -6.2534 & 11.428 & 1.587 & 34.42 & 81 & 4.02 & 4.5 \\
\hline 1837 & $18503724-0614364$ & 282.6552 & -6.2434 & 12.001 & 1.422 & 35.28 & 131 & 7.89 & 10.5 \\
\hline 1658 & $18504563-0612038$ & 282.6901 & -6.2011 & 11.622 & 1.475 & 35.13 & 111 & 5.40 & 7.0 \\
\hline 1625 & $18504737-0617184$ & 282.6974 & -6.2884 & 11.652 & 1.729 & 32.01 & 102 & 3.84 & 4.5 \\
\hline 1446 & $18505494-0616182$ & 282.7289 & -6.2717 & 11.860 & 1.468 & 34.76 & 104 & 5.24 & 7.0 \\
\hline 1423 & $18505581-0618148$ & 282.7325 & -6.3041 & 11.414 & 1.627 & 35.23 & 121 & 4.73 & 6.0 \\
\hline 1364 & $18505755-0613461$ & 282.7398 & -6.2295 & 11.830 & 1.211 & 30.60 & 162 & 9.14 & 11.5 \\
\hline 1286 & $18505944-0612435$ & 282.7477 & -6.2121 & 11.872 & 1.398 & 34.38 & 117 & 8.25 & 11.0 \\
\hline 1256 & $18510023-0616594$ & 282.7510 & -6.2832 & 11.586 & 1.733 & 35.23 & 88 & 3.54 & 5.0 \\
\hline 1248 & $18510032-0617183$ & 282.7513 & -6.2884 & 12.081 & 1.461 & 35.23 & 122 & 6.48 & 9.0 \\
\hline 1184 & $18510200-0617265$ & 282.7583 & -6.2907 & 11.426 & 1.604 & 32.03 & 81 & 3.68 & 4.5 \\
\hline 1145 & $18510289-0615301$ & 282.7620 & -6.2584 & 12.014 & 1.453 & 32.76 & 118 & 5.43 & 7.0 \\
\hline 1117 & $18510341-0616202$ & 282.7642 & -6.2723 & 11.801 & 1.438 & 36.14 & 105 & 8.03 & 10.0 \\
\hline 1111 & $18510358-0616112$ & 282.7649 & -6.2698 & 11.902 & 1.418 & 34.63 & 113 & 5.31 & 7.5 \\
\hline 1090 & $18510399-0620414$ & 282.7666 & -6.3448 & 11.872 & 1.566 & 34.01 & 102 & 4.65 & 6.0 \\
\hline 963 & $18510662-0612442$ & 282.7776 & -6.2123 & 11.720 & 1.510 & 33.23 & 113 & 5.04 & 7.0 \\
\hline 916 & $18510786-0617119$ & 282.7828 & -6.2866 & 11.621 & 1.463 & 33.86 & 188 & 7.62 & 9.5 \\
\hline 899 & $18510833-0616532$ & 282.7847 & -6.2814 & 11.736 & 1.466 & 33.14 & 94 & 4.64 & 5.5 \\
\hline 827 & $18511013-0615486$ & 282.7922 & -6.2635 & 11.493 & 1.503 & 36.88 & 115 & 3.51 & 4.5 \\
\hline 816 & $18511048-0615470$ & 282.7937 & -6.2631 & 11.627 & 1.507 & 33.08 & 121 & 7.45 & 9.0 \\
\hline 779 & $18511116-0614340$ & 282.7965 & -6.2428 & 11.468 & 1.732 & 33.42 & 87 & 4.61 & 5.5 \\
\hline 686 & $18511452-0616551$ & 282.8105 & -6.2820 & 11.923 & 1.480 & 35.15 & 97 & 7.27 & 9.0 \\
\hline 669 & $18511534-0618359$ & 282.8139 & -6.3100 & 11.974 & 1.553 & 33.75 & 161 & 7.13 & 9.0 \\
\hline 660 & $18511571-0618146$ & 282.8155 & -6.3041 & 11.807 & 1.490 & 35.17 & 139 & 4.58 & 6.0 \\
\hline 411 & $18512662-0614537$ & 282.8609 & -6.2483 & 11.559 & 1.669 & 33.90 & 128 & 5.41 & 6.5 \\
\hline 160 & $18514034-0617128$ & 282.9181 & -6.2869 & 11.525 & 1.539 & 33.25 & 121 & 5.26 & 7.0 \\
\hline 136 & $18514130-0620125$ & 282.9221 & -6.3368 & 11.849 & 1.499 & 33.21 & 83 & 2.84 & 4.0 \\
\hline
\end{tabular}

Notes. ID and photometric data were taken from Sung et al. (1999); ${ }^{(a)}$ values from the GES database; ${ }^{(b)}$ determined in this work.

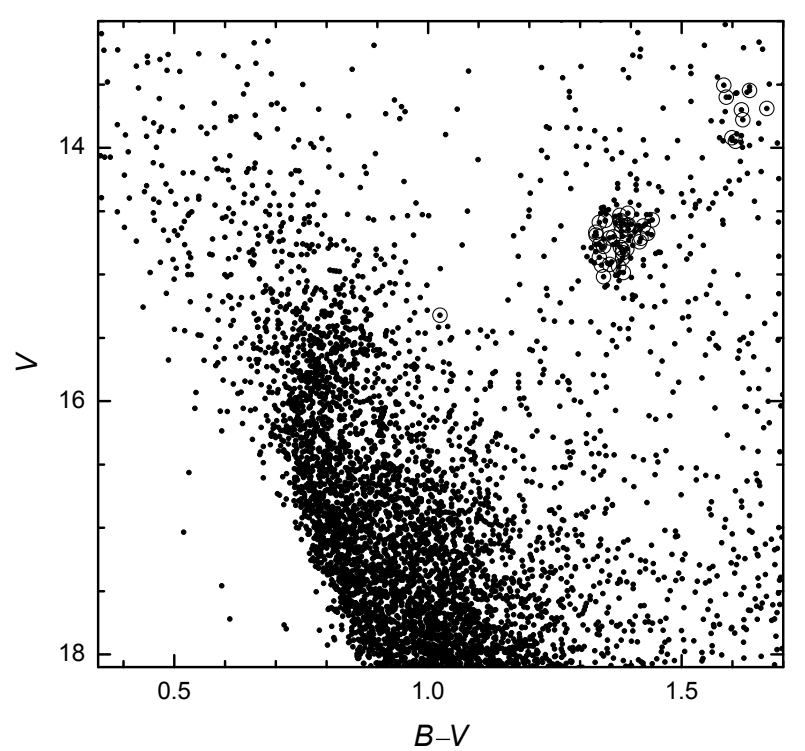

Fig. 1. Colour-magnitude diagram of the open cluster Trumpler 20. The stars investigated in this work are indicated by circles. The diagram is based on photometry by Carraro et al. (2010).

all observing runs (see Sacco et al. 2014, for more details). A correction for telluric contamination is not yet implemented, so none is applied before co-addition. This may cause a problem in some cases, as we encountered for the [O I] $6300.3 \AA$ line in stars of Trumpler 20, because of the systemic radial velocity of that cluster. The oxygen line in the spectra of NGC 4815 and NGC 6705 stars was not affected by telluric lines.

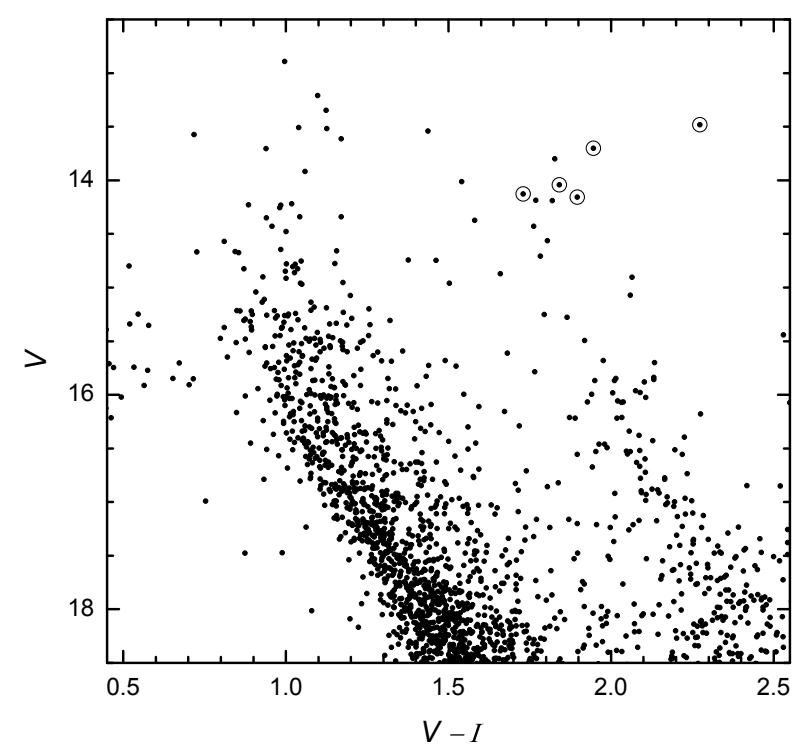

Fig. 2. Colour-magnitude diagram of the open cluster NGC 4815. The stars investigated in this work are indicated by circles. The diagram is based on photometry by Prisinzano et al. (2001).

\subsection{Main atmospheric parameters}

The main atmospheric parameters of the stars were determined spectroscopically using a technique described by Smiljanic et al. (2014). To make full use of the available expertise of the consortium, all the spectra were analysed in parallel by 13 nodes of scientists. The methodology and codes used by each Node are described in detail by Smiljanic et al. (2014). A number of 


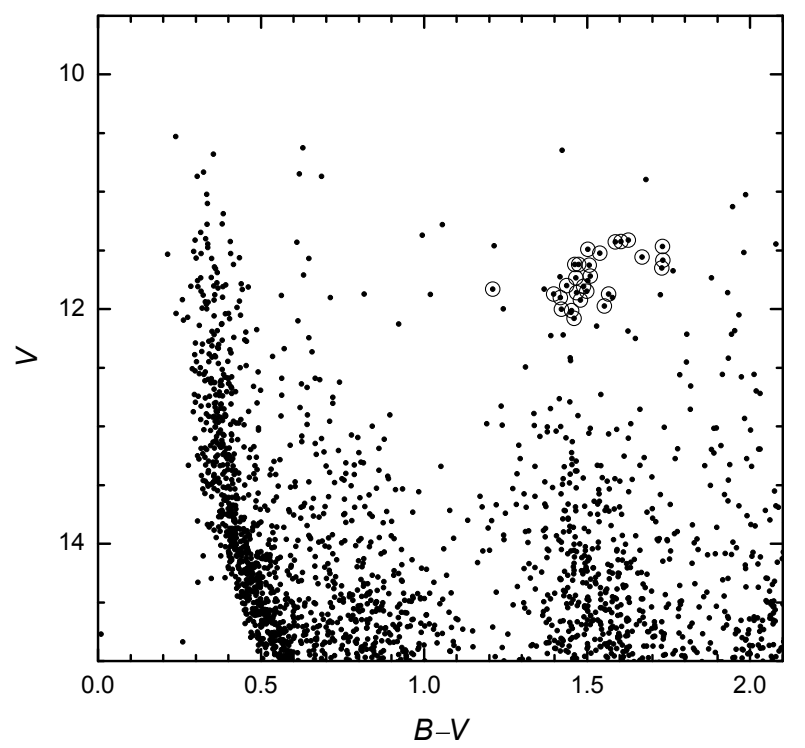

Fig. 3. Colour-magnitude diagram of the open cluster NGC 6705. The stars investigated in this work are indicated by circles. The diagram is based on photometry by Sung et al. (1999).

constraints have been imposed on input data used in the analysis to guarantee some degree of homogeneity in the final results: the use of a common line list, the use of one single set of model atmospheres, and the analysis of common calibration targets. The Gaia-ESO Survey line list version 4.0 was used to determine the main atmospheric parameters of the cluster stars (Heiter et al., in prep.). For model atmospheres, the MARCS grid (Gustafsson et al. 2008) was adopted. The grid consists of spherically-symmetric models complemented by plane-parallel models for stars of high surface gravity (between $\log g=3.0$ and 5.0, or 5.5 for cooler models). The models are based on hydrostatic equilibrium, LTE, and the mixing-length theory of convection. The assumed solar abundances are those of Grevesse et al. (2007). Thus, when metallicities in the format $[\mathrm{El} / \mathrm{H}]$ or $[\mathrm{El} / \mathrm{Fe}]^{3}$ are quoted in this work, the solar elemental abundances are the following: $A(\mathrm{Fe})_{\odot}=7.45, A(\mathrm{C})_{\odot}=8.39, A(\mathrm{~N})_{\odot}=7.78$, and $A(\mathrm{O})_{\odot}=8.66$.

To homogenise the results of different nodes and quantify the method-to-method dispersion of each parameter the median and the associated median absolute deviation (MAD) have been used. The first step was a zeroth-order quality control of the results of each node (values with very large error bars were removed). Then, we used results of the benchmark stars to weight the performance of each node in different regions of the parameter space. Finally, the weighted-median value of the validated results was adopted as the recommended value of that parameter. For the Internal Data Release 2 (GESviDR2Final) results, used in this work, the median of the method-to-method dispersion is $55 \mathrm{~K}, 0.13 \mathrm{dex}$, and $0.07 \mathrm{dex}$ for $T_{\mathrm{eff}}, \log g$, and $[\mathrm{Fe} / \mathrm{H}]$, respectively.

\section{3. $C, N$, and $O$ abundances}

Abundances of carbon, nitrogen, and oxygen were determined by the GES Vilnius node using the spectral synthesis with the code BSYN, developed at the Uppsala Astronomical Observatory. The $\mathrm{C}_{2}$ Swan $(1,0)$ band head at $5135 \AA$ and

\footnotetext{
3 We use the customary spectroscopic notation $[X / Y] \equiv$ $\log _{10}\left(N_{X} / N_{Y}\right)_{\text {star }}-\log _{10}\left(N_{X} / N_{Y}\right)_{\odot}$.
}

Table 4. Effects on derived abundances, $\Delta[\mathrm{El} / \mathrm{H}]$, resulting from model changes for the star NGC 67051625.

\begin{tabular}{lrrccc}
\hline \hline Species & $\begin{array}{r}\Delta T_{\text {eff }} \\
\pm 100 \mathrm{~K}\end{array}$ & $\begin{array}{r}\Delta \log g \\
\pm 0.3\end{array}$ & $\begin{array}{c}\Delta v_{\mathrm{t}} \\
\pm 0.3 \mathrm{~km} \mathrm{~s}^{-1}\end{array}$ & $\begin{array}{c}\Delta[\mathrm{Fe} / \mathrm{H}] \\
\pm 0.1\end{array}$ & Total \\
\hline $\mathrm{C}\left(\mathrm{C}_{2}\right)$ & 0.03 & 0.05 & 0.00 & 0.05 & 0.07 \\
$\mathrm{~N}(\mathrm{CN})$ & 0.07 & 0.13 & 0.01 & 0.11 & 0.18 \\
$\mathrm{O}([\mathrm{O} \mathrm{I}])$ & 0.01 & 0.12 & 0.00 & 0.01 & 0.12 \\
\hline
\end{tabular}

$\mathrm{C}_{2}$ Swan $(0,1)$ band head at $5635.5 \AA$ were investigated in order to determine the carbon abundance. The $\mathrm{C}_{2}$ bands are suitable for carbon abundance investigations since yield give the same carbon abundances as [C I] lines, which are not sensitive to non-local thermodynamical equilibrium (NLTE) deviations (cf. Clegg et al. 1981; Gustafsson et al. 1999).

The interval $6470-6490 \AA$ containing ${ }^{12} \mathrm{C}^{14} \mathrm{~N}$ bands was used for the nitrogen abundance analysis. The oxygen abundance was determined from the forbidden [OI] line at $6300.31 \AA$. Following Johansson et al. (2003), we took into account the oscillator strength values for ${ }^{58} \mathrm{Ni}$ and ${ }^{60} \mathrm{Ni}$, which blend the oxygen line. Lines of $[\mathrm{OI}]$ are considered as very good indicators of oxygen abundances. It was determined that they are not only insensitive to NLTE effects, but also give similar oxygen abundance results with 3D and 1D model atmospheres (cf. Asplund et al. 2004; Pereira et al. 2009).

All the synthetic spectra have been calibrated to the solar spectrum by Kurucz (2005) to make the analysis strictly differential. Figures 5-8 display examples of spectrum syntheses for the programme stars. The best-fit abundances were determined by eye.

In fitting the observed spectra with theoretical spectra the stellar rotation was taken into account. Approximate values of stellar rotation velocities were evaluated for the Survey stars as described by Sacco et al. (2014). Values of $v \sin i$ were calculated using an empirical relation of a full width half maximum of the the cross- correlation function $\left(\mathrm{CCF}_{\mathrm{FWHM}}\right)$ and $v \sin i$, which was specifically derived for this project. Accuracy of those values depends on the spectral type of a star. Since the values of $v \sin i$, provided by the Gaia-ESO Survey in GESviDR2Final, were estimated before the main atmospheric parameters of stars were known, consequently $v \sin i$ can be improved after the stellar parameters are determined. Thus we did that for the investigated stars using stronger surrounding lines in spectral regions around the investigated $\mathrm{C}, \mathrm{N}$, and $\mathrm{O}$ features. The initial and updated $v \sin i$ values are presented in the last two columns of Tables 1 to 3 . The new values on average are larger by about $1.4 \pm 0.7 \mathrm{~km} \mathrm{~s}^{-1}$. We did not need an extremely high accuracy of the $v \sin i$ values since their influence in determining of the $\mathrm{C}, \mathrm{N}$, and $\mathrm{O}$ abundances is not crucial (see Sect. 2.4). However, especially for stars of our youngest open cluster NGC 6705 , rotating up to $11 \mathrm{~km} \mathrm{~s}^{-1}$, a higher accuracy of elemental abundances was certainly achieved.

\subsection{Evaluation of uncertainties}

The sensitivity of the abundance estimates $\Delta[\mathrm{El} / \mathrm{H}]$ to changes in the atmospheric parameters are listed for the star NGC 67051625 in Table 4. Clearly, the abundances are not much affected when the parameter uncertainties quoted at the end of Sect. 2.2 are considered.

Since abundances of $\mathrm{C}, \mathrm{N}$, and $\mathrm{O}$ are bound together by the molecular equilibrium in the stellar atmosphere, we 


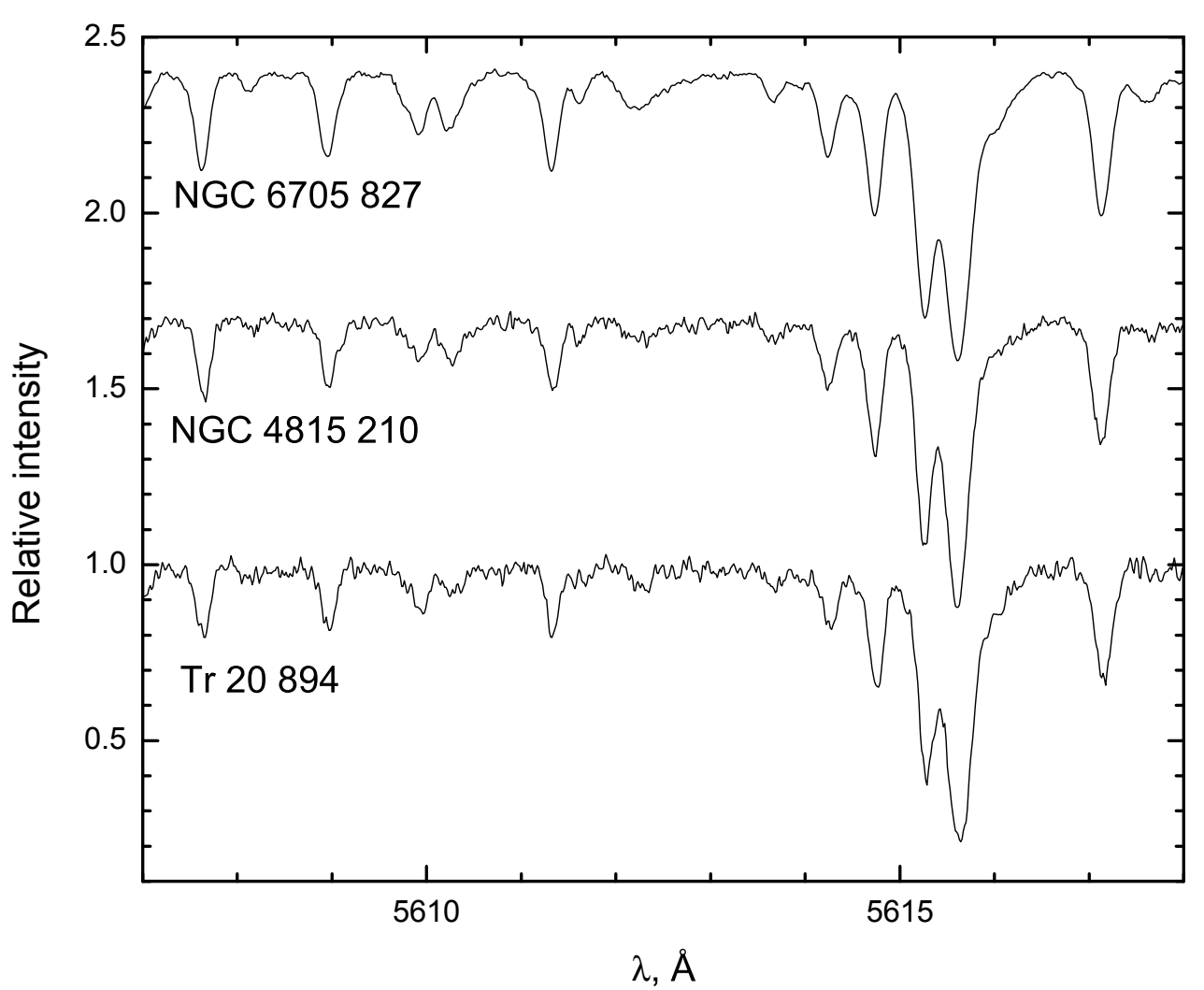

Fig. 4. Examples of stellar spectra for our programme stars. An offset of 0.7 in relative flux is applied for clarity.

also investigated how an error in one of them typically affects the abundance determination of another. Thus $\Delta[\mathrm{O} / \mathrm{H}]=$ 0.10 causes $\Delta[\mathrm{C} / \mathrm{H}]=0.05$ and $\Delta[\mathrm{N} / \mathrm{H}]=0.12 ; \Delta[\mathrm{C} / \mathrm{H}]=0.10$ causes $\Delta[\mathrm{N} / \mathrm{H}]=-0.17$ and $\Delta[\mathrm{O} / \mathrm{H}]=0.02 ; \Delta[\mathrm{N} / \mathrm{H}]=0.10$ has no effect on either the carbon or the oxygen abundances.

Random errors of abundance determinations, in this study mainly caused by uncertainties of the continuum placement and $\mathrm{S} / \mathrm{N}$, can be evaluated from the scatter of abundances determined from different lines. The mean value of $[\mathrm{C} / \mathrm{H}]$ scatter from the $\mathrm{C}_{2} 5135$ and $5635.5 \AA$ lines in Trumpler 20 is equal to $\pm 0.03 \mathrm{dex}$, in NGC $4815 \pm 0.06 \mathrm{dex}$, and in NGC 6705 $\pm 0.02 \mathrm{dex}$. The same low mean values of scatter are found in the case of $[\mathrm{N} / \mathrm{H}]$ as well. Since oxygen abundances were determined just from one line at $6300.3 \AA$, our ad hoc evaluation of random errors for $[\mathrm{O} / \mathrm{H}]$ is $\pm 0.05 \mathrm{dex}$ in NGC 6705 , and slightly larger, of about \pm 0.15 dex, in NGC 4815 .

The influence of stellar rotation on the $\mathrm{C}, \mathrm{N}$, and $\mathrm{O}$ abundances in slowly rotating stars of our sample is very small; it becomes more important in stars rotating more rapidly. If $v \sin i$ is about $3 \mathrm{~km} \mathrm{~s}^{-1}$, a difference of $\pm 1 \mathrm{~km} \mathrm{~s}^{-1}$ changes the abundances only by \pm 0.01 dex. If $v \sin i$ is about $7 \mathrm{~km} \mathrm{~s}^{-1}$, a difference of $\pm 1 \mathrm{~km} \mathrm{~s}^{-1}$ changes the abundances of nitrogen and oxygen by about \pm 0.06 dex, while carbon abundances change only by \pm 0.02 dex.

\section{Results and discussion}

The atmospheric parameters $T_{\text {eff }}, \log g, v_{t},[\mathrm{Fe} / \mathrm{H}]$ and abundances of $\mathrm{C}, \mathrm{N}$, and $\mathrm{O}$ chemical elements relative to iron $[\mathrm{El} / \mathrm{Fe}]$ of the programme stars in Trumpler 20, NGC 4815, and NGC 6705 are presented in Tables 5-7, respectively. They display the GESviDR2Final results.

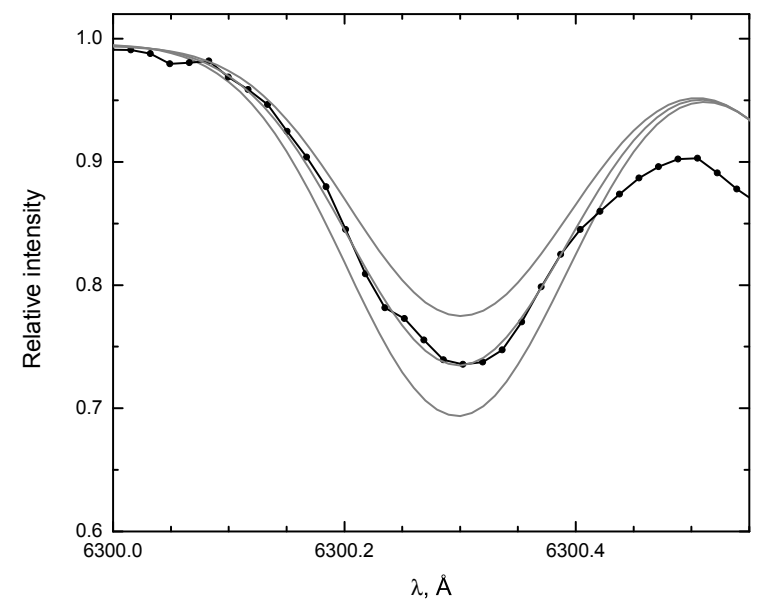

Fig. 5. A fit to the forbidden [OI] line at $6300.3 \AA$ in the programme star NGC 6705779 . The observed spectrum is shown as a black line with dots. The synthetic spectra with $[\mathrm{O} / \mathrm{Fe}]=0.07 \pm 0.1$ are shown as grey lines.

The abundances of oxygen were not determined for stars in Trumpler 20 because of blending by telluric lines. When determining abundances of carbon and nitrogen for stars of this cluster, we adopted a value of -0.05 dex for $[\mathrm{O} / \mathrm{Fe}]$. At the metallicity of this cluster, which is $[\mathrm{Fe} / \mathrm{H}]=0.10 \pm 0.08$ (s.d.) as determined from 42 Trumpler 20 stars in the GESviDR2Final (Table 5), this $[\mathrm{O} / \mathrm{Fe}]$ value within about \pm 0.05 dex uncertainty is typical (cf. Pagel \& Tautvaišiene 1995; Bensby et al. 2014). A lower mean $[\mathrm{O} / \mathrm{Fe}]$ value $(-0.18 \mathrm{dex})$, based on an analysis of five clump stars, was presented for this cluster by Carraro et al. (2014) even though the mean $[\mathrm{Fe} / \mathrm{H}]$ value $(0.09 \pm 0.10 \mathrm{dex})$ is very close to ours. We decided not to use this rather low $[\mathrm{O} / \mathrm{Fe}]$ 
G. Tautvaišienė et al.: The Gaia-ESO Survey: CNO abundances in the open clusters Trumpler 20, NGC 4815, and NGC 6705

Table 5. Main atmospheric parameters and elemental abundances for stars in the open cluster Trumpler 20.

\begin{tabular}{|c|c|c|c|c|c|c|c|}
\hline GES ID & $\begin{array}{c}T_{\text {eff }} \\
\mathrm{K}\end{array}$ & $\log g$ & $\begin{array}{c}v_{t} \\
\mathrm{~km} \mathrm{~s}^{-1} \\
\end{array}$ & {$[\mathrm{Fe} / \mathrm{H}]$} & {$[\mathrm{C} / \mathrm{H}]$} & {$[\mathrm{N} / \mathrm{H}]$} & $\mathrm{C} / \mathrm{N}$ \\
\hline $12383595-6045245$ & 5006 & 3.11 & 1.43 & 0.18 & -0.04 & 0.46 & 1.26 \\
\hline $12383659-6045300$ & 4863 & 2.84 & 1.23 & 0.21 & 0.04 & 0.60 & 1.10 \\
\hline $12385807-6030286$ & 4488 & 2.13 & 1.48 & 0.00 & -0.20 & 0.39 & 1.02 \\
\hline $12390411-6034001$ & 4494 & 2.17 & 1.49 & 0.02 & -0.19 & 0.43 & 0.95 \\
\hline $12390478-6041475$ & 5017 & 2.99 & 1.39 & 0.13 & -0.11 & 0.46 & 1.07 \\
\hline $12390710-6038057$ & 5007 & 3.07 & 1.20 & 0.08 & -0.17 & 0.53 & 0.79 \\
\hline $12391004-6038402$ & 4516 & 2.17 & 1.57 & -0.04 & -0.24 & 0.39 & 0.93 \\
\hline $12391114-6036527$ & 5003 & 3.09 & 1.34 & 0.20 & -0.02 & 0.51 & 1.17 \\
\hline $12391201-6036322$ & 4990 & 3.12 & 1.35 & 0.20 & -0.01 & 0.54 & 1.12 \\
\hline $12391577-6034406$ & 4873 & 2.99 & 1.37 & -0.03 & -0.18 & 0.35 & 1.17 \\
\hline $12392585-6038279$ & 5086 & 3.24 & 1.39 & 0.14 & -0.08 & 0.56 & 0.91 \\
\hline $12392637-6040217$ & 4967 & 2.90 & 1.45 & 0.06 & -0.09 & 0.54 & 0.93 \\
\hline $12392700-6036053$ & 4830 & 2.70 & 1.46 & 0.06 & -0.11 & 0.46 & 1.07 \\
\hline $12393132-6039422$ & 5000 & 3.09 & 1.39 & 0.11 & -0.09 & 0.60 & 0.81 \\
\hline $12393741-6032568$ & 5030 & 3.10 & 1.38 & 0.14 & -0.09 & 0.47 & 1.10 \\
\hline $12393782-6039051$ & 4931 & 3.00 & 1.43 & 0.08 & -0.10 & 0.56 & 0.87 \\
\hline $12394051-6041006$ & 4976 & 2.94 & 1.42 & 0.15 & -0.09 & 0.54 & 0.93 \\
\hline $12394122-6040040$ & 5033 & 3.09 & 1.40 & 0.14 & -0.09 & 0.53 & 0.95 \\
\hline $12394309-6039193$ & 4889 & 2.92 & 1.39 & 0.13 & -0.13 & 0.51 & 0.91 \\
\hline $12394387-6033166$ & 5029 & 3.05 & 1.49 & 0.14 & -0.09 & 0.63 & 0.76 \\
\hline $12394419-6034412$ & 4990 & 3.03 & 1.40 & 0.17 & -0.13 & 0.57 & 0.79 \\
\hline $12394475-6038339$ & 4895 & 2.86 & 1.43 & 0.09 & -0.10 & 0.58 & 0.83 \\
\hline $12394517-6038257$ & 4955 & 3.00 & 1.36 & 0.22 & -0.02 & 0.55 & 1.07 \\
\hline $12394596-6038389$ & 4936 & 2.95 & 1.42 & 0.06 & -0.11 & 0.44 & 1.12 \\
\hline $12394690-6033540$ & 4984 & 2.97 & 1.42 & 0.08 & -0.10 & 0.51 & 0.98 \\
\hline $12394715-6040584$ & 4570 & 2.20 & 1.57 & -0.05 & -0.27 & 0.44 & 0.78 \\
\hline $12394742-6038411$ & 4918 & 2.92 & 1.47 & 0.12 & -0.06 & 0.57 & 0.93 \\
\hline $12394899-6033282$ & 4919 & 2.78 & 1.49 & 0.05 & -0.12 & 0.52 & 0.91 \\
\hline $12395426-6038369$ & 4949 & 2.95 & 1.41 & 0.17 & -0.09 & 0.54 & 0.93 \\
\hline $12395555-6037268$ & 4923 & 2.93 & 1.34 & 0.11 & -0.09 & 0.51 & 1.00 \\
\hline 12395569-6035233* & 5954 & 3.65 & 1.50 & 0.12 & 0.35 & & \\
\hline $12395655-6039011$ & 4918 & 2.91 & 1.36 & 0.13 & -0.09 & 0.44 & 1.17 \\
\hline $12395712-6039335$ & 4964 & 3.00 & 1.40 & 0.09 & -0.08 & 0.49 & 1.07 \\
\hline $12395975-6035072$ & 4898 & 2.89 & 1.42 & 0.12 & -0.06 & 0.57 & 0.93 \\
\hline 12400111-6031395 & 4910 & 2.99 & 1.37 & 0.13 & -0.10 & 0.49 & 1.02 \\
\hline $12400116-6035218$ & 4879 & 2.94 & 1.40 & 0.14 & -0.11 & 0.41 & 1.20 \\
\hline $12400260-6039545$ & 4503 & 2.12 & 1.53 & -0.03 & -0.22 & 0.36 & 1.05 \\
\hline $12400278-6041192$ & 4993 & 3.13 & 1.33 & 0.19 & -0.02 & 0.57 & 1.02 \\
\hline $12400451-6036566$ & 4369 & 2.06 & 1.51 & -0.05 & -0.27 & 0.38 & 0.89 \\
\hline $12400755-6035445$ & 4431 & 2.08 & 1.55 & 0.01 & -0.20 & 0.43 & 0.93 \\
\hline $12402229-6037419$ & 4969 & 2.95 & 1.46 & 0.09 & -0.09 & 0.52 & 0.98 \\
\hline $12402480-6043101$ & 4570 & 2.24 & 1.57 & -0.04 & -0.25 & 0.38 & 0.93 \\
\hline $\begin{array}{c}\text { Mean } \\
\text { s.d. }\end{array}$ & & & & $\begin{array}{r}0.10 \\
\pm 0.08\end{array}$ & $\begin{array}{l}-0.10 \\
\pm 0.10\end{array}$ & $\begin{array}{r}0.50 \\
\pm 0.07\end{array}$ & $\begin{array}{c}0.98 \\
\pm 0.12\end{array}$ \\
\hline
\end{tabular}

Notes. ${ }^{(*)}$ This star is a subgiant located close to the main sequence turn-off and had no CN bands strong enough for the nitrogen abundance determination.

Table 6. Main atmospheric parameters and elemental abundances for stars in the open cluster NGC 4815.

\begin{tabular}{ccccrcrrr}
\hline \hline GES ID & $\begin{array}{c}T_{\text {eff }} \\
\mathrm{K}\end{array}$ & $\log g$ & $\begin{array}{c}v_{t} \\
\mathrm{~km} \mathrm{~s}^{-1}\end{array}$ & {$[\mathrm{Fe} / \mathrm{H}]$} & {$[\mathrm{C} / \mathrm{H}]$} & {$[\mathrm{N} / \mathrm{H}]$} & {$[\mathrm{O} / \mathrm{H}]$} & $\mathrm{C} / \mathrm{N}$ \\
\hline $12572442-6455173$ & 4328 & 1.88 & 1.67 & 0.01 & -0.14 & 0.62 & 0.11 & 0.69 \\
$12574328-6457386$ & 4905 & 2.56 & 1.89 & -0.07 & -0.19 & 0.51 & 0.16 & 0.79 \\
$12575511-6458483$ & 4964 & 2.76 & 1.59 & 0.02 & -0.07 & 0.57 & 0.22 & 0.91 \\
$12575529-6456536$ & 5062 & 2.68 & 1.13 & -0.04 & -0.29 & 0.43 & -0.02 & 0.76 \\
$12580262-6456492$ & 4966 & 2.52 & 1.72 & 0.01 & -0.17 & 0.52 & 0.13 & 0.81 \\
\hline Mean & & & & -0.01 & -0.17 & 0.53 & 0.12 & 0.79 \\
s.d. & & & & \pm 0.04 & \pm 0.08 & \pm 0.07 & \pm 0.09 & \pm 0.08 \\
\hline
\end{tabular}

Notes. ${ }^{(*)}$ This value was not included into the mean $[\mathrm{O} / \mathrm{Fe}]$ value of the cluster stars. 
A\&A 573, A55 (2015)

Table 7. Main atmospheric parameters and elemental abundances for stars in the open cluster NGC 6705.

\begin{tabular}{ccccrrrrr}
\hline \hline GES ID & $\begin{array}{c}T_{\text {eff }} \\
\mathrm{K}\end{array}$ & $\log g$ & $\begin{array}{c}v_{t} \\
\mathrm{~km} \mathrm{~s}^{-1}\end{array}$ & {$[\mathrm{Fe} / \mathrm{H}]$} & {$[\mathrm{C} / \mathrm{H}]$} & {$[\mathrm{N} / \mathrm{H}]$} & {$[\mathrm{O} / \mathrm{H}]$} & $\mathrm{C} / \mathrm{N}$ \\
\hline $18502831-0615122$ & 4543 & 1.82 & 1.83 & -0.05 & -0.14 & 0.57 & 0.04 & 0.78 \\
$18503724-0614364$ & 4889 & 2.73 & 1.68 & 0.07 & 0.01 & 0.74 & 0.27 & 0.74 \\
$18504563-0612038$ & 4635 & 2.14 & 1.62 & -0.07 & -0.12 & 0.56 & 0.13 & 0.83 \\
$18504737-0617184$ & 4330 & 1.71 & 1.64 & -0.01 & -0.10 & 0.65 & 0.06 & 0.71 \\
$18505494-0616182$ & 4639 & 2.24 & 1.55 & -0.05 & -0.16 & 0.54 & 0.08 & 0.79 \\
$18505581-0618148$ & 4496 & 1.94 & 1.73 & 0.07 & 0.04 & 0.54 & 0.16 & 1.26 \\
$18505755-0613461$ & 4828 & 2.55 & 1.40 & 0.02 & -0.14 & 0.53 & 0.07 & 0.85 \\
$18505944-0612435$ & 4883 & 2.43 & 1.61 & 0.01 & -0.12 & 0.65 & 0.11 & 0.68 \\
$18510023-0616594$ & 4351 & 1.85 & 1.75 & -0.04 & -0.03 & 0.50 & 0.14 & 1.17 \\
$18510032-0617183$ & 4867 & 2.39 & 1.73 & 0.07 & -0.05 & 0.66 & 0.15 & 0.78 \\
$18510200-0617265$ & 4321 & 1.80 & 1.60 & -0.01 & -0.01 & 0.42 & 0.11 & 1.48 \\
$18510289-0615301$ & 4751 & 2.35 & 1.52 & 0.00 & -0.09 & 0.55 & 0.12 & 0.91 \\
$18510341-0616202$ & 4798 & 2.16 & 1.89 & 0.06 & -0.05 & 0.66 & 0.14 & 0.78 \\
$18510358-0616112$ & 4810 & 2.31 & 1.77 & 0.02 & -0.07 & 0.59 & 0.18 & 0.87 \\
$18510399-0620414$ & 4660 & 2.09 & 1.71 & -0.03 & -0.12 & 0.59 & 0.08 & 0.78 \\
$18510662-0612442$ & 4698 & 2.07 & 1.80 & -0.02 & -0.09 & 0.55 & 0.12 & 0.91 \\
$18510786-0617119$ & 4767 & 2.48 & 1.87 & 0.06 & 0.03 & 0.70 & 0.25 & 0.85 \\
$18510833-0616532$ & 4713 & 2.19 & 1.83 & 0.05 & -0.04 & 0.69 & 0.17 & 0.74 \\
$18511013-0615486$ & 4540 & 2.09 & 1.54 & -0.07 & -0.16 & 0.65 & 0.14 & 0.62 \\
$18511048-0615470$ & 4688 & 2.06 & 1.81 & -0.14 & -0.17 & 0.59 & 0.06 & 0.69 \\
$18511116-0614340$ & 4332 & 1.74 & 1.67 & 0.01 & -0.08 & 0.67 & 0.08 & 0.71 \\
$18511452-0616551$ & 4730 & 2.25 & 1.86 & -0.04 & -0.10 & 0.53 & 0.16 & 0.93 \\
$18511534-0618359$ & 4675 & 2.18 & 1.85 & 0.08 & -0.05 & 0.64 & 0.13 & 0.81 \\
$18511571-0618146$ & 4706 & 2.11 & 1.73 & 0.06 & -0.08 & 0.64 & 0.10 & 0.76 \\
$18512662-0614537$ & 4422 & 1.97 & 1.62 & 0.03 & -0.06 & 0.69 & 0.11 & 0.71 \\
$18514034-0617128$ & 4653 & 2.02 & 1.75 & 0.01 & -0.12 & 0.65 & 0.13 & 0.68 \\
$18514130-0620125$ & 4687 & 2.13 & 1.73 & 0.01 & -0.10 & 0.64 & 0.12 & 0.72 \\
\hline Mean & & & & 0.00 & -0.08 & 0.61 & 0.13 & 0.83 \\
s.d. & & & & \pm 0.05 & \pm 0.06 & \pm 0.07 & \pm 0.05 & \pm 0.19 \\
\hline
\end{tabular}

value from Carraro et al. (2014) since the surface gravity $\log g$ values for the clump stars in their work are systematically lower than our values by about 0.3 dex. This difference in $\log g$ may cause the difference in oxygen abundances of about 0.12 dex (see Table 4).

Preliminary analysis and interpretation of $\alpha$ - and iron-peakelement abundances in these three open clusters have been made by Magrini et al. (2014) on the basis of GESviDR1Final. It was concluded that the three clusters are essentially homogeneous in all investigated elements. As follows from our study, this conclusion is also valid for abundances of carbon, nitrogen, and oxygen. Tables 5-7 also contain mean values of $[\mathrm{Fe} / \mathrm{H}]$ and $[\mathrm{El} / \mathrm{Fe}]$, and it is seen that standard deviations (s.d.) do not exceed \pm 0.08 dex.

A sample of stars in Trumpler 20 contains one subgiant located close to the main sequence turn-off (Fig. 1), i.e. Trumpler 20430 (GES ID 12395569-6035233). Only the carbon abundance was determined for this star from quite weak $\mathrm{C}_{2}$ lines. All $\mathrm{CN}$ features were too weak for the nitrogen abundance determination. The abundance of carbon is by 0.45 dex larger in this unevolved star than in the investigated giants. It is expected that carbon abundances in unevolved stars are larger than in evolved, however, this large value of carbon in Trumpler 20430 is probably not of primordial origin.

The new mean GESviDR2Final $[\mathrm{Fe} / \mathrm{H}]$ values for the investigated clusters agree with the GESviDR1Final results within the uncertainties. An average iron abundance of 12 members of Trumpler $20[\mathrm{Fe} / \mathrm{H}]=0.17 \pm 0.03$ (Donati et al. 2014) is superceded by $[\mathrm{Fe} / \mathrm{H}]=0.10 \pm 0.08$ based on 42 stars. In NGC 4815, a mean $[\mathrm{Fe} / \mathrm{H}]$ of NGC 4815 was $+0.03 \pm 0.05 \mathrm{dex}$ (Friel et al. 2014). For the same five stars, the GESviDR2Final results yielded $[\mathrm{Fe} / \mathrm{H}]=-0.01 \pm 0.04$ dex. An average iron abundance of 21 NGC 6705 members in the GESviDR1Final was $[\mathrm{Fe} / \mathrm{H}]=0.10 \pm 0.06$. A larger sample of 27 NGC 6705 stars in GESviDR2Final gives $[\mathrm{Fe} / \mathrm{H}]=0.0 \pm 0.05$. The new values were determined using advanced, in comparison to iDR 1, atmospheric parameter homogenisation procedures (see Smiljanic et al. 2014 for details). The mean iron abundance of $10 \mathrm{~K}$-giants in NGC 6705, determined from high-resolution spectroscopy by Gonzalez \& Wallerstein (2000), $0.07 \pm 0.05$ dex is close to our result. Eight of those stars are present in our sample.

\subsection{Carbon and nitrogen}

Star clusters are recognised as the optimum test case for judging stellar evolutionary models and the validity of their physical assumptions. In our work, we will use the determined carbon and nitrogen abundance ratios for this purpose. It is well known that canonical models, in which convection is the only driver of mixing inside a stellar interior, explain observations of stars in the lower part of the giant branch only. Low- and intermediatemass stars during the subsequent ascent on the RGB exhibit signatures of complex physical processes of extra-mixing that require challenging modelling. In order to describe the observed surface abundances in different types of stars, various mechanisms of extra-mixing were proposed by a number of scientific groups (see e.g. reviews by Chanamé et al. 2005; Charbonnel 2006, and papers by Charbonnel \& Lagarde 2010; Denissenkov 2010; Lagarde et al. 2011, 2012; Palmerini et al. 2011a; Wachlin et al. 2011; Angelou et al. 2012; Karakas \& Lattanzio 2014, and references therein). 


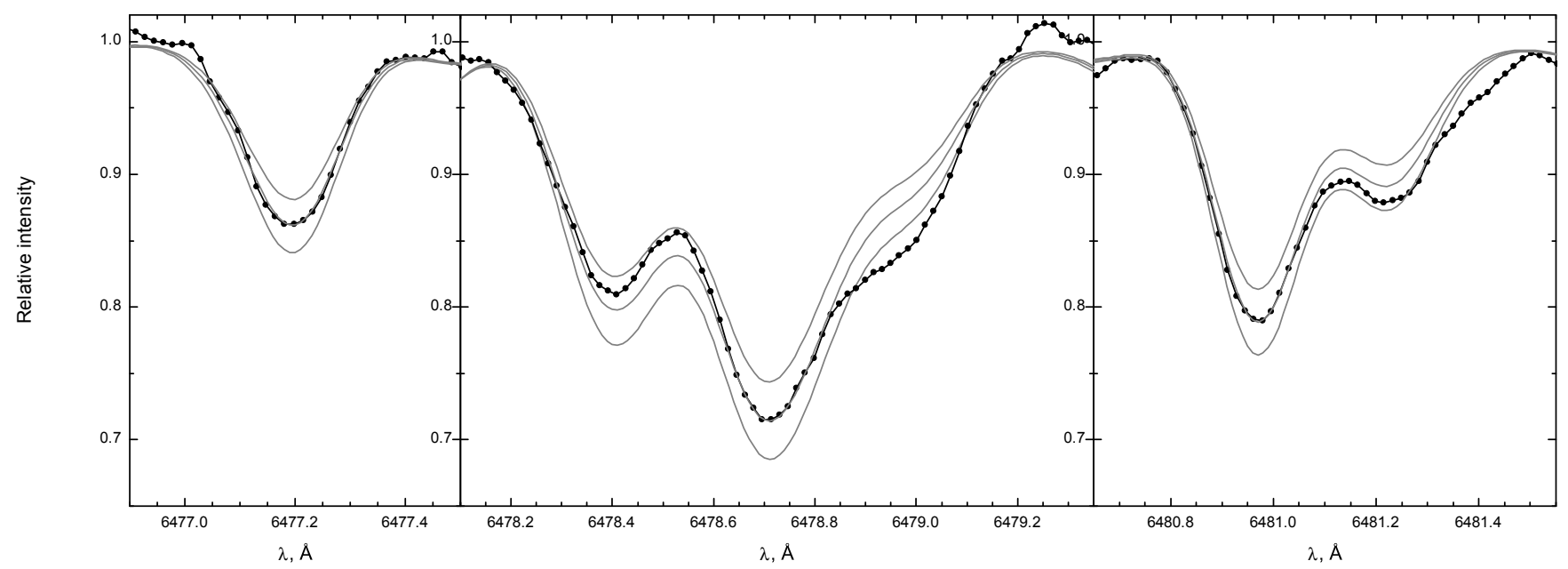

Fig. 6. A fit to the CN bands in the programme star NGC 6705 1625. The observed spectrum is shown as a black line with dots. The synthetic spectra with $[\mathrm{N} / \mathrm{Fe}]=0.66 \pm 0.1$ are shown as grey lines.

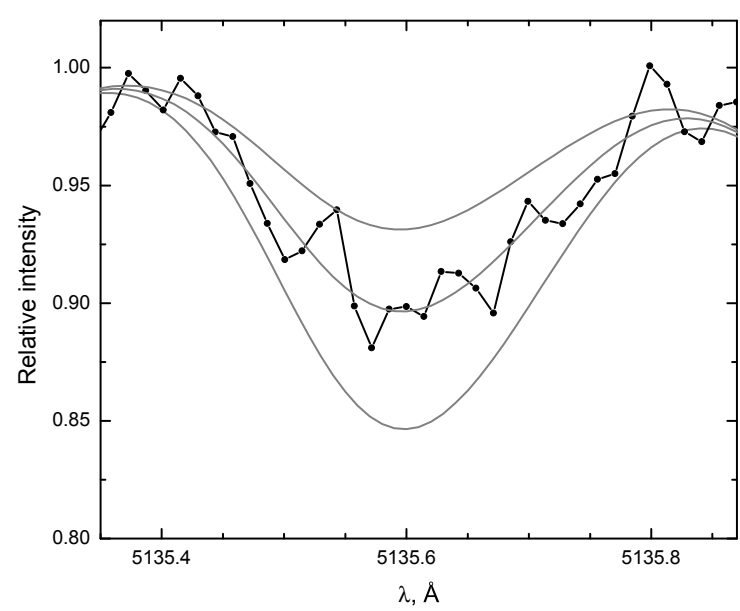

Fig. 7. A fit to the $\mathrm{C}_{2}$ Swan $(1,0)$ band head at $5135 \AA$ in the programme star $\operatorname{Tr} 2063$. The observed spectrum is shown as a black line with dots. The synthetic spectra with $[\mathrm{C} / \mathrm{Fe}]=-0.22 \pm 0.1$ are shown as grey lines.

The newest models with extra-mixing include a thermohaline instability induced mixing based on ideas of Eggleton et al. (2006) and Charbonnel \& Zahn (2007). Eggleton et al. (2006) found a mean molecular weight $(\mu)$ inversion in their $1 M_{\odot}$ stellar evolution model, occurring after the luminosity bump on the RGB, when the hydrogen burning shell reaches the chemically homogeneous part of the envelope. The $\mu$-inversion is produced by the reaction ${ }^{3} \mathrm{He}\left({ }^{3} \mathrm{He}, 2 \mathrm{p}\right){ }^{4} \mathrm{He}$, as predicted by Ulrich (1972). It does not occur earlier, because the magnitude of the $\mu$-inversion is small and negligible compared to a stabilizing $\mu$-stratification. Following Eggleton et al., Charbonnel \& Zahn (2007) computed stellar models including the prescription by Ulrich (1972) and extended them to the case of a non-perfect gas for the turbulent diffusivity produced by that instability in a stellar radiative zone. They found that a double diffusive instability referred to as thermohaline convection, which had been discussed long ago in the literature (Stern 1960), is important in the evolution of red giants. This mixing connects the convective envelope with the external wing of the hydrogen burning shell and induces surface abundance modifications in red giant stars.

Quantitative abundance values of mixing-sensitive chemical elements based on the thermohaline mixing model have been

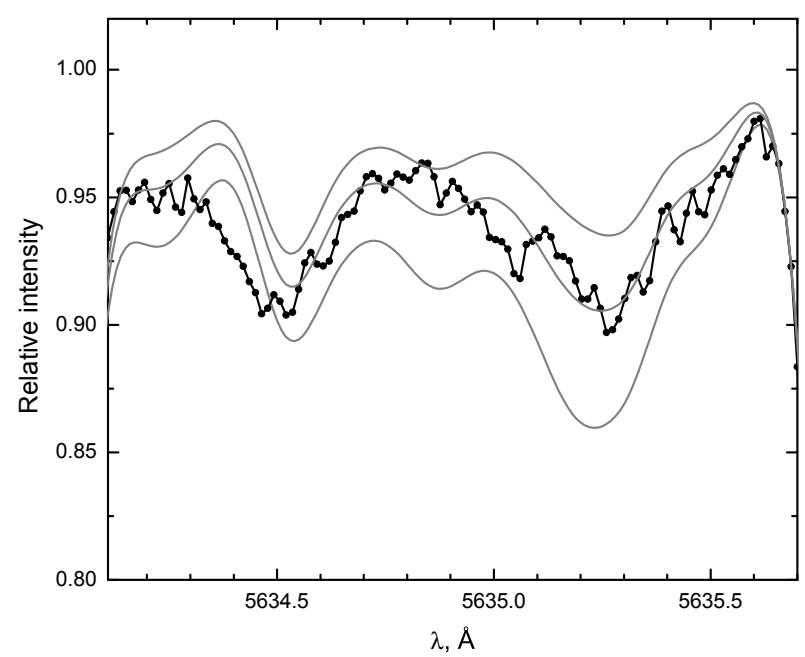

Fig. 8. A fit to the $C_{2}$ Swan $(0,1)$ band head at $5635.5 \AA$ in the programme star NGC 4815358 . The observed spectrum is shown as a black line with dots. The synthetic spectra with $[\mathrm{C} / \mathrm{Fe}]=-0.16 \pm 0.1$ are shown as grey lines.

provided by Charbonnel \& Lagarde (2010). In Fig. 9 we plotted a trend of the 1 st dredge-up $\mathrm{C} / \mathrm{N}$ values computed using the standard (1DUP ST) model, as well as the trend of thermohaline induced extra-mixing (TH). The thermohaline mixing could be an important physical process governing the surface $\mathrm{C} / \mathrm{N}$ ratios of stars with initial masses below $1.5 M_{\odot}$, and its efficiency is increasing with decreasing initial stellar mass. The turn-off masses of the open clusters in our work are quite large (1.9, 2.6, and 3.3 $M_{\odot}$ in Trumpler 20, NGC 4815, and NGC 6705, respectively). In comparison with the models of the 1st dredge-up and the thermohaline mixing by Charbonnel \& Lagarde (2010), which are indistinguishable in this interval of stellar masses, the mean $\mathrm{C} / \mathrm{N}$ ratios of the investigated clusters lie slightly lower, yet agree with them within the quoted uncertainties.

A decrease of $\mathrm{C} / \mathrm{N}$ values can also be caused by stellar rotation. Charbonnel \& Lagarde (2010) computed models of rotation-induced mixing for stars at the zero-age main sequence (ZAMS) having rotational velocities of $110 \mathrm{~km} \mathrm{~s}^{-1}$, $250 \mathrm{~km} \mathrm{~s}^{-1}$, and $300 \mathrm{~km} \mathrm{~s}^{-1}$. The convective envelope was supposed to rotate as a solid body through the evolution. The transport coefficients for chemicals associated with 


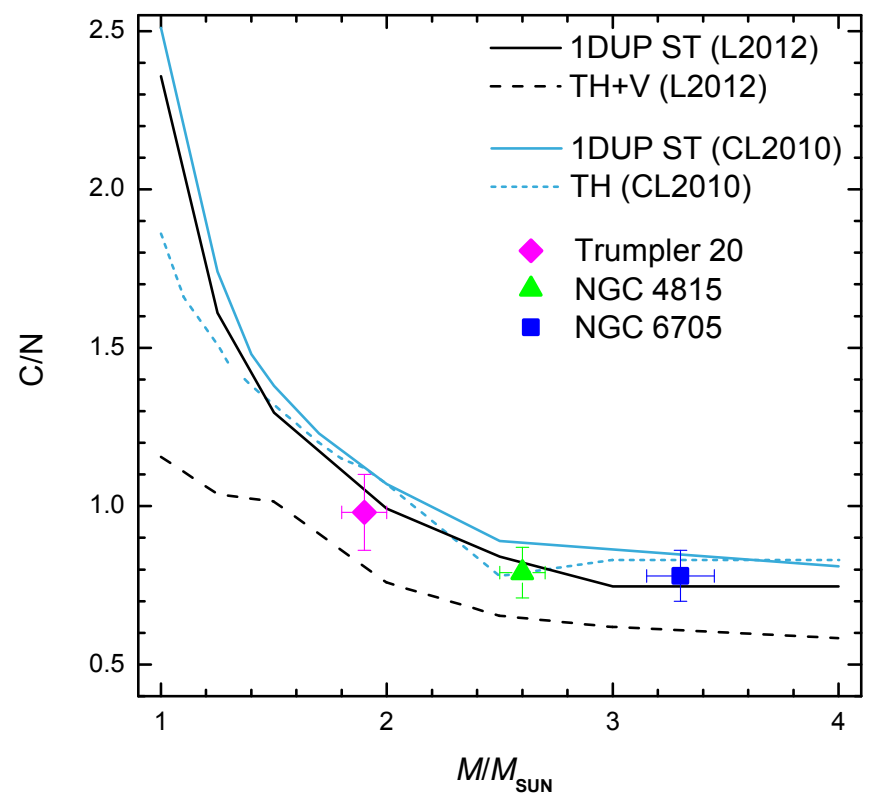

Fig. 9. Mean carbon-to-nitrogen ratios in stars of open clusters as a function of stellar turn-off mass. The diamond represents Trumpler 20 stars, the triangle is for NGC 4815, and the square is for NGC 6705 stars. The solid lines represent the $\mathrm{C} / \mathrm{N}$ ratios predicted for stars at the first dredge-up with standard stellar evolutionary models by Charbonnel \& Lagarde (2010; blue upper line) and, more recently, Lagarde et al. (2012; black lower line). The blue dashed line shows the prediction when just thermohaline extra-mixing is introduced (Charbonnel \& Lagarde 2010), and the black dashed line is for the model that includes both the thermohaline and rotation induced mixing (Lagarde et al. 2012), see Sect. 3.1 for more explanations.

thermohaline- and rotation-induced mixing were simply added in the diffusion equation and the possible interactions between the two mechanisms were not considered. The rotation-induced mixing modifies the internal chemical structure of main sequence stars, although its signatures are revealed only later in the stellar evolution when the first dredge-up occurs. More recently, Lagarde et al. (2012) computed models with both the thermohaline and rotation induced mixing acting together. In Fig. 9 we show their model computed with standard (ST) prescriptions, as well as the model including both thermohaline convection and rotation-induced mixing (TH+V). Lagarde et al. (2012) also assumed solid-body rotation in the convective regions, however, in addition they assumed that the transport of angular momentum is dominated by the large amount of turbulence in these regions which instantaneously flattens out the angular velocity profile as it does for the abundance profiles. The initial rotation velocity of the models on the ZAMS was chosen at $45 \%$ of the critical velocity at that point and leads to mean velocities on the main sequence between 90 and $137 \mathrm{~km} \mathrm{~s}^{-1}$. In Fig. 9, we can see that the $\mathrm{C} / \mathrm{N}$ values in Trumpler 20, NGC 4815, and NGC 6705 stars are not decreased as much as the model predicts if both the thermohaline and rotation induced extra mixing is at work. Indeed, the observed $\mathrm{C} / \mathrm{N}$ ratios are very close to predictions of the standard model at first dredge-up. In the already mentioned analysis of ten NGC 6705 stars by Gonzalez \& Wallerstein (2000), carbon isotope ratios were also determined. All the giants have ${ }^{12} \mathrm{C} /{ }^{13} \mathrm{C} \approx 20$, which also agrees with the 1 st dredge-up model.

The thermohaline induced extra-mixing theory is under development. Magnetic activity also may play a role. Denissenkov et al. (2009) investigated a heat exchange between rising magnetic flux rings and their surrounding medium and proposed a model of magneto-thermohaline mixing. On the basis of three-dimensional numerical simulations of thermohaline convection, Denissenkov \& Merryfield (2011) suggested that the salt-finger ${ }^{4}$ spectrum might be shifted towards larger diameters by the toroidal magnetic field. Nucci \& Busso (2014) investigated magnetic advection as a mechanism for deep mixing. According to their evaluation, in this case the mixing velocities are smaller than for convection, but larger than for diffusion and adequate for extra mixing in red giants. Unfortunately, these studies have not provided values of $\mathrm{C} / \mathrm{N}$ that we could compare with observations.

Wachlin et al. (2011) computed full evolutionary sequences of RGB stars close to the luminosity bump and found that thermohaline mixing is not efficient enough for fingering convection to reach the bottom of the convective envelope of red giants. In order to reach the contact, the diffusion coefficient has to be artificially increased by about four orders of magnitude.

A much larger, homogeneous data-base of CNO abundances in open clusters will be released in the framework of the Gaia-ESO Survey collaboration, which will significantly constrain mixing mechanisms in extant stellar evolutionary models. Unfortunately, carbon isotope ratios will not be investigated in this survey since there are no suitable spectral features in the selected spectral regions.

\subsection{Oxygen}

As described in the previous section, the abundances of $\mathrm{C}$ and $\mathrm{N}$ of the stars analysed in this work have been modified by stellar evolution processes and, hence, do not trace the initial composition of the stars anymore. The abundances of $\mathrm{O}$, instead, still reflect the chemical composition of the stars at birth and can, thus, be used in studies of Galactic chemical evolution.

In Magrini et al. (2014), several abundance ratios $([\mathrm{Mg} / \mathrm{Fe}]$, $[\mathrm{Si} / \mathrm{Fe}],[\mathrm{Ca} / \mathrm{Fe}],[\mathrm{Ti} / \mathrm{Fe}],[\mathrm{Cr} / \mathrm{Fe}],[\mathrm{Ni} / \mathrm{Fe}])$ measured in the same clusters (GESviDR1Final) were compared with the predictions of two chemical evolution models (Magrini et al. 2009; Romano et al. 2010) and with field star abundance data. This comparison hinted at an inner birthplace for NGC 6705. For NGC 4815, the $[\mathrm{Mg} / \mathrm{Fe}]$ ratio was also higher and similar to that in NGC 6705 . Here, we compare the $[\mathrm{O} / \mathrm{Fe}]$ abundance ratios measured in NGC 4815 and NGC 6705 with the predictions of the same models (Figs. 10 and 11).

In Fig. 10, we show the comparison with the $[\mathrm{O} / \mathrm{Fe}]$ versus $[\mathrm{Fe} / \mathrm{H}]$ trends predicted by Magrini et al. (2009) and by Romano et al. (2010) for different Galactocentric radii $\left(R_{\mathrm{GC}}\right.$ equal to 4,6 , and $8 \mathrm{kpc})$. The data are consistent, within the errors, with the history of chemical enrichment of the solar neighbourhood and of the inner disc $\left(R_{\mathrm{GC}} \leq 4 \mathrm{kpc}\right)$. The trends predicted by Romano et al. (2010; Fig. 10, right panel) are almost independent of the Galactocentric radius, because of the adoption of an efficiency of star formation constant with the Galactocentric distance in their model. The trends by Magrini et al. (2009; Fig. 10, left panel) vary with the Galactocentric distance as a result of an adopted radial dependence of the star formation efficiency and of the infall rate.

4 The expression "salt-finger" comes from oceanology where thermohaline mixing is also widely used to model the regions of cooler, less salty water below the warmer water where the salinity is higher because of the evaporation from the surface. The so-called "fingers" of the warmer water penetrate the cooler water and the mixing occurs when the heat excess is exchanged (e.g., Schmitt 2003; Ruddick 2003; Kunze 2003; Radko 2010). 


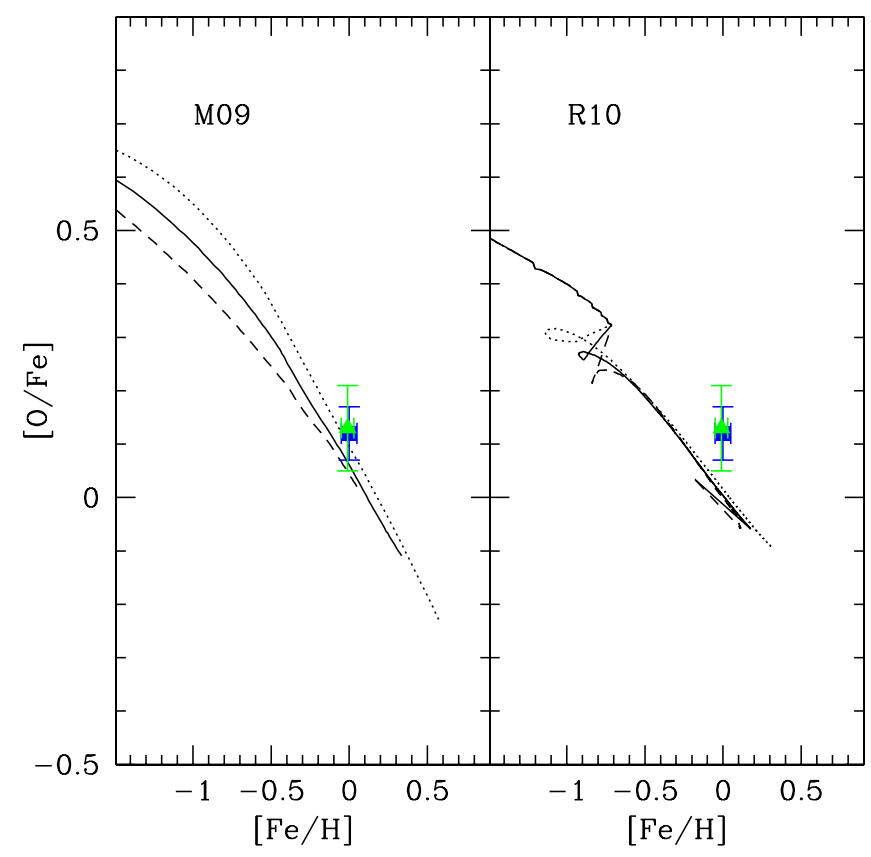

Fig. 10. $[\mathrm{O} / \mathrm{Fe}]$ vs. $[\mathrm{Fe} / \mathrm{H}]$ in open clusters: NGC 4815 (triangle) and NGC 6705 (square). The curves are the theoretical predictions at three $R_{\mathrm{GC}}: 4 \mathrm{kpc}$ (dotted line), $6 \mathrm{kpc}$ (continuous line), and $8 \mathrm{kpc}$ (dashed line), for the model of Magrini et al. (2009, M09; left panel) and the model by Romano et al. (2010, R10; right panel). The theoretical ratios are normalised to the solar abundances predicted by each model.

The age of the clusters is hidden in this comparison, so in Fig. 11, we show the radial gradients of $[\mathrm{O} / \mathrm{Fe}]$ and $[\mathrm{O} / \mathrm{H}]$ at three epochs: the present time, $1 \mathrm{Gyr}$ ago, and $3 \mathrm{Gyr}$ ago, i.e. in an age range comparable with the ages of the three clusters ( 0.3, $\sim 0.5$ Gyr and $~ 1.5$ Gyr for NGC 6705, NGC 4815, and $\operatorname{Tr} 20$, respectively). Oxygen abundances are in good agreement with both models, while $[\mathrm{O} / \mathrm{Fe}]$ is slightly higher than the model predictions.

The comparison with the $[\mathrm{O} / \mathrm{Fe}]$ versus $[\mathrm{Fe} / \mathrm{H}]$ trends predicted by Magrini et al. (2009) for different Galactocentric radii ( $R_{\mathrm{GC}}$ equal to 4,6 , and $8 \mathrm{kpc}$; Fig. 10, left panel) seems to suggest an inner origin for both clusters. The curve for $R_{\mathrm{GC}}=4 \mathrm{kpc}$ corresponds to a stronger infall rate and a higher star formation efficiency than assumed for $R_{\mathrm{GC}}=8 \mathrm{kpc}$. Thus, if one interprets the agreement between model predictions and observations as an indication of the conditions, in terms of star formation and infall rate, of the interstellar medium from which the clusters were born, he/she is led to conclude that their high $[\mathrm{O} / \mathrm{Fe}]$ for their $[\mathrm{Fe} / \mathrm{H}]$ indicate that they were born in a place subject to a more rapid enrichment than the solar neighbourhood, i.e. the inner disc. However, being more conservative, we notice that the data are not inconsistent, within the errors, with the history of chemical enrichment of the solar neighbourhood. The trends predicted by Romano et al. (2010; Fig. 10, right panel) are almost independent of the Galactocentric radius because of the adoption of an efficiency of star formation constant with the Galactocentric distance in their model.

It is worth stressing at this point that the chemical evolution models adopted in this work do not take stellar migration into account. It is, however, unlikely that the predictions about the evolution of the heavy elements discussed in this paper are significantly modified by the process of stellar migration, since the parent stars would not travel very large distances before releasing their products, assuming typical

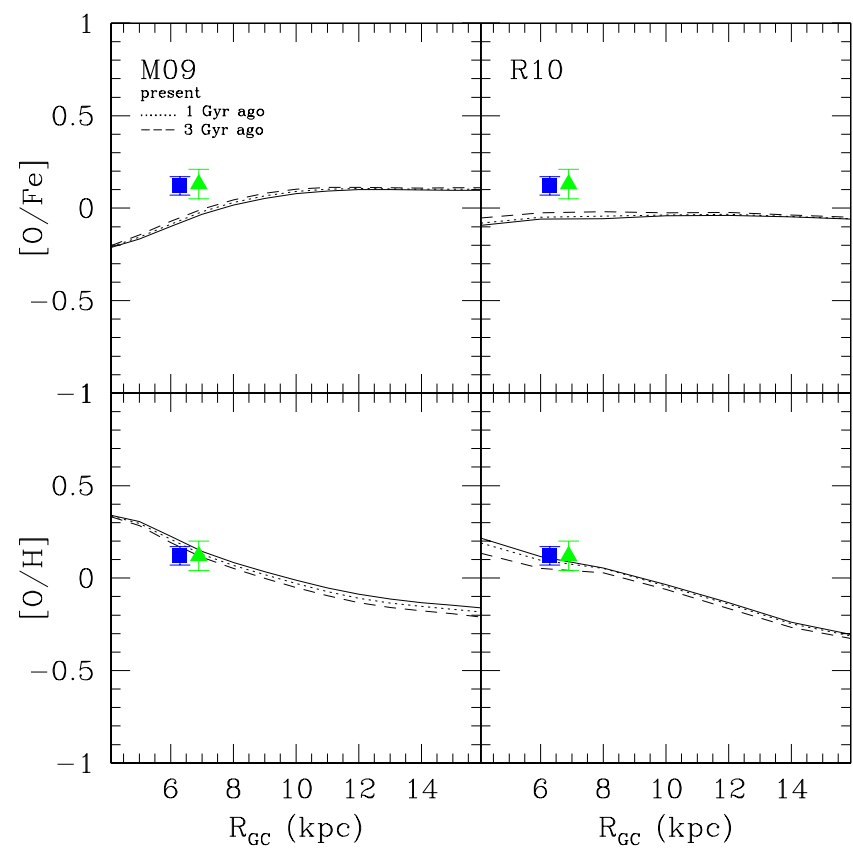

Fig. 11. $[\mathrm{O} / \mathrm{Fe}]$ and $[\mathrm{O} / \mathrm{H}]$ vs. $R_{\mathrm{GC}}$ in open clusters: NGC 4815 (triangle) and NGC 6705 (square). The curves are the theoretical predictions at three epochs: present time (continuous line), $1 \mathrm{Gyr}$ ago (dotted line), and 3 Gyr ago (dashed line) for the model of Magrini et al. (2009, M09; left panel) and the model by Romano et al. (2010, R10; right panel). The theoretical ratios are normalised to the solar abundances predicted by each model.

velocities of $1 \mathrm{~km} \mathrm{~s}^{-1}\left(\sim 1 \mathrm{kpc} \mathrm{Gyr}^{-1}\right.$; Kordopatis et al. 2013) for the stars.

The oxygen abundance results obtained in this work for NGC 4815 and NGC 6705 will be utilised also for other aims of the Galactic evolution studies later on when a larger number of GES open clusters will be investigated.

\section{Conclusions}

In this paper, we present the analysis of $\mathrm{C}, \mathrm{N}$, and $\mathrm{O}$ abundances in three open clusters observed in the Gaia-ESO Survey: Trumpler 20, NGC 6705, and for the first time in NGC 4815.

The C/N ratios in Trumpler 20, NGC 4815, and NGC 6705 stars, which have turn-off masses of about 1.9, 2.6, and $3.3 M_{\odot}$, are $\mathrm{C} / \mathrm{N}=0.98 \pm 0.12$ (s.d.), $\mathrm{C} / \mathrm{N}=0.79 \pm 0.08$, and $\mathrm{C} / \mathrm{N}=$ $0.83 \pm 0.19$, respectively. The $\mathrm{C} / \mathrm{N}$ values in the investigated clusters are not as low as predicted if thermohaline instability and rotation-induced mixing are at work in stars as suggested by Lagarde et al. (2012); rather, they are consistent with the predictions of the standard models from the same authors, or with models with only thermohaline-induced mixing as in Charbonnel \& Lagarde (2010).

We compare $[\mathrm{O} / \mathrm{H}]$ and $[\mathrm{O} / \mathrm{Fe}]$ abundance ratios with the results of two chemical evolution models: Magrini et al. (2009), and Romano et al. (2010). The former includes a radial variation of the star formation efficiency and infall rate, while the latter assumes a constant star formation efficiency across the disc. The average values of $[\mathrm{O} / \mathrm{Fe}]$ vs. $[\mathrm{Fe} / \mathrm{H}]$ in NGC 6705 and NGC 4815 are consistent, within the errors, with the clusters being born where they are found now in the disc. However, a comparison with the temporal evolution of the radial gradients of $[\mathrm{O} / \mathrm{Fe}]$ and $[\mathrm{O} / \mathrm{H}]$ show that they are slightly higher in $[\mathrm{O} / \mathrm{Fe}]$ than expected 
for their age and present location. The $[\mathrm{Mg} / \mathrm{Fe}]$ ratio in these clusters is also enhanced (Magrini et al. 2014).

Many more open clusters will be investigated in the GaiaESO Survey, thus providing the homogeneous observational data needed to further develop both stellar and Galactic evolutionary models.

Acknowledgements. Based on data products from observations made with ESO Telescopes at the La Silla Paranal Observatory under programme ID 188.B-3002. This work was partly supported by the European Union FP7 programme through ERC grant number 320360 and by the Leverhulme Trust through grant RPG-2012-541. We acknowledge the support from INAF and Ministero dell' Istruzione, dell' Università e della Ricerca (MIUR) in the form of the grant "Premiale VLT 2012" and "The Chemical and Dynamical Evolution of the Milky Way and Local Group Galaxies" (prot. 2010LY5N2T). The results presented here benefit from discussions held during the Gaia-ESO workshops and conferences supported by the ESF (European Science Foundation) through the GREAT Research Network Programme. T. Bensby was funded by grant No. 621-20093911 from the Swedish Research Council. T. Morel acknowledges financial support from Belspo for contract PRODEX Gaia-DPAC. R. Smiljanic was supported by the National Science Center of Poland through grant 2012/07/B/ST9/04428. This research has made use of the SIMBAD database, operated at CDS, Strasbourg, France, of NASA's Astrophysics Data System, of the compilation of atomic lines from the Vienna Atomic Line Database (VALD), and of the WEBDA database, operated at the Department of Theoretical Physics and Astrophysics of the Masaryk University.

\section{References}

Angelou, G. C., Stancliffe, R. J., Church, R. P., Lattanzio, J. C., \& Smith, G. H. 2012, ApJ, 749, 128

Asplund, M., Grevesse, N., Sauval, A. J., Allende Prieto, C., \& Kiselman, D. 2004, A\&A, 417, 751

Bensby, T., Feltzing, S., \& Oey, M. S. 2014, A\&A, 562, A71

Boothroyd, A. I., \& Sackmann, I.-J. 1999, ApJ, 510, 232

Bragaglia, A., Sestito, P., Villanova, S., et al. 2008, A\&A, 480, 79

Busso, M., Wasserburg, G. J., Nollett, K. M., \& Calandra, A. 2007, ApJ, 671, 802

Cameron L. M. 1985, A\&A, 147, 39

Cantat-Gaudin, T., Vallenari, A., Zaggia, S., et al. 2014, A\&A, 569, A17

Cantiello, M., \& Langer, N. 2010, A\&A, 521, A9

Carraro, G., Ng, Y. K., \& Portinari, L. 1998, MNRAS, 296, 1045

Carraro, G., Geisler, D., Villanova, S., Frinchaboy, P. M., \& Majewski, S. R. 2007, A\&A, 476, 217

Carraro, G., Costa, E., \& Ahumada, J. A. 2010, AJ, 140, 954

Carraro, G., Villanova, S., Monaco, L., et al. 2014, A\&A, 562, A39

Chanamé, J., Pinsonneault, M., \& Terndrup, D. M. 2005, ApJ, 631, 540

Charbonnel, C. 1994, A\&A, 282, 811

Charbonnel, C. 2006, EAS Pub. Ser., 19, 125

Charbonnel, C., \& Lagarde, N. 2010, A\&A, 522, A10

Charbonnel, C., \& Zahn, J.-P. 2007, A\&A, 467, L15

Charbonnel, C., Brown, J. A., \& Wallerstein, G. 1998, A\&A, 332, 204

Chen, L., Hou, J. L., \& Wang, J. J. 2003, AJ, 125, 1397

Church, R. P., Lattanzio, J., Angelou, G., Tout, C. A., \& Stancliffe, R. J. 2014, MNRAS, 443, 977

Clegg, R. E. S., Tomkin, J., \& Lambert, D. L. 1981, ApJ, 250, 262

Dearborn, D. S. P., Eggleton, P. P., \& Schramm, D. A. 1976, ApJ, 203, 445

Dekker, H., D’Odorico, S., Kaufer, A., Delabre, B., \& Kotzlowski, H. 2000, in

Optical and IR Telescope Instrumentation and Detectors, eds. M. Iye, \& A. F.

Moorwood, SPIE Conf. Ser., 4008, 534

Denissenkov, P. A. 2010, ApJ, 723, 563

Denissenkov, P. A., \& Merryfield, W. J. 2011, ApJ, 727, L8

Denissenkov, P. A., Pinsonneault, M., \& MacGregor, K. B. 2009, ApJ, 696, 1823

Donati, P., Cantat Gaudin, T., Bragaglia, A., et al. 2014, A\&A, 561, A94

Eggleton, P. P., Dearborn, D. S. P., \& Lattanzio, J. C. 2006, Science, 314, 1580

Friel, E. D. 1995, ARA\&A, 33, 381

Friel, E. D., Janes K. A., Tavarez, M., et al. 2002, AJ, 124, 2693

Friel, E. D., Donati, P., Bragaglia, A., et al. 2014, A\&A, 563, A117

Gilmore, G., Randich, S., Asplund, M., et al. 2012, The Messenger, 147, 25

Gilroy K. K. 1989, ApJ, 347, 835

Gilroy K. K., \& Brown J. A. 1991, ApJ, 371, 578

Gonzalez, G., \& Wallerstein, G. 2000, PASP, 112, 774

Gratton, R. G., Sneden, C., Carretta, E., \& Bragaglia, A. 2000, A\&A, 345, 169

Grevesse, N., Asplund, M., \& Sauval, A. J. 2007, Space Sci. Rev., 130, 105

Gustafsson, B., Karlsson, T., Olsson, E., Edvardsson, B., \& Ryde, N. 1999, A\&A, 342, 426

Gustafsson, B., Edvardsson, B., Eriksson, K., et al. 2008, A\&A, 486, 951
Iben, I., Jr. 1965, ApJ, 142, 1447

Iben, I., Jr. 1967, ApJ, 147, 624

Jacobson, H. R., Friel, E. D., \& Pilachowski, C. A. 2009, AJ, 137, 4753

Janes, K. A. 1979, ApJS, 39, 135

Johansson, S., Litzén, U., Lundberg, H., \& Zhang, Z. 2003, ApJ, 584, L107

Karakas, A. I., \& Lattanzio, J. C. 2014, PASA, 31, e030

Kordopatis, G., Gilmore, G., Steinmetz, M., et al. 2013, AJ, 146, 134

Kunze, E. 2003, Progress in Oceanography, 56, 399

Kurucz, R. L. 2005, Mem. S. A. It. Supp., 8, 189

Lada, C. J., \& Lada, E. A. 2003, ARA\&A, 41, 57

Lagarde, N., Charbonnel, C., Decressin, T., \& Hagelberg, J. 2011, A\&A, 536, A28

Lagarde, N., Decressin, T., Charbonnel, C., et al. 2012, A\&A, 543, A108

Lépine, J. R. D., Cruz, P., Scarano, S., et al. 2011, MNRAS, 417, 698

Luck R. E. 1994, ApJS, 91, 309

Magrini, L., Sestito, P., Randich, S., \& Galli, D. 2009, A\&A, 494, 95

Magrini, L., Randich, S., \& Zoccali, M. 2010, A\&A, 523, A11

Magrini, L., Randich, S., \& Romano, D. 2014, A\&A, 563, A44

Maeder, A., Meynet, G., Lagarde, N., \& Charbonnel, C. 2013, A\&A, 553, A1

Mikolaitis, Š., Tautvaišienė, G., Gratton, R., Bragaglia, A., \& Carretta, E. 2010, MNRAS, 407, 1866

Mikolaitis, S., Tautvaišienè, G., Gratton, R., Bragaglia, A., \& Carretta, E. 2011a, MNRAS, 413, 2199

Mikolaitis, Š., Tautvaišienè, G., Gratton, R., Bragaglia, A., \& Carretta, E. 2011b, MNRAS, 416, 1092

Mikolaitis, Š., Tautvaišienè, G., Gratton, R., Bragaglia, A., \& Carretta, E. 2012, A\&A, 541, A137

Nucci, M. C., \& Busso, M. 2014, ApJ, 787, 141

Origlia, L., Valenti, E., Rich, R. M., \& Ferraro, F. R. 2006, AJ, 646, 499

Pagel, B. E. J., \& Tautvaišienè, G. 1995, MNRAS, 276, 505

Panagia, N., \& Tosi, M. 1980, A\&A, 81, 375

Pallavicini, R. 2003, Mem. S. A. It. Supp., 3, 74

Palmerini, S., La Cognata, M., Cristallo, S., \& Busso, M. 2011a, ApJ, 729, 3

Palmerini, S., Cristallo, S., Busso, M., et al. 2011b, ApJ, 741, 26

Pancino, E., Carrera, R., Rossetti, E., \& Gallart, C. 2010, A\&A, 511, A56

Pasquini, L., Avila, G., Blecha, A., et al. 2002, The Messenger, 110, 1

Pereira, T. M. D., Asplund, M., \& Kiselman, D. 2009, A\&A, 508, 1403

Prisinzano, L., Carraro, G., Piotto, G., et al. 2001, A\&A, 369, 851

Radko, T. 2010, J. Fluid Mech., 645, 121

Randich, S., \& Gilmore, G. 2013, The Messenger, 154, 47

Romano, D., Karakas, A. I., Tosi, M., \& Matteucci, F. 2010, A\&A, 522, A32

Ruddick, B. 2003, Progress in Oceanography, 56, 483

Sacco, G. G., Morbidelli, L., Franciosini, E., et al. 2014, A\&A, 565, A113

Salaris, M., Weiss, A., \& Percival, S. M. 2004, A\&A, 414, 163

Schmitt, R. 2003, Progress in Oceanography, 56, 419

Sestito, P., Bragaglia, A., Randich, S., et al. 2008, A\&A, 488, 943

Smiljanic, R., Gauderon, R., North, P., et al. 2009, A\&A, 502, 267

Smiljanic, R., Korn, A. J., Bergemann, M., et al. 2014, A\&A, 570, A22

Stern, M. E. 1960, Tellus, 12, 172

Spitoni, E., Romano, D., Matteucci, F., \& Ciotti, L. 2014 [arXiv: 1407. 5797]

Sung, H., Bessell, M. S., Lee, H.-W., Kang, Y. H., \& Lee, S.-W. 1999, MNRAS, 310,982

Tautvaišiene, G., Edvardsson, B., Tuominen, I., \& Ilyin, I. 2000, A\&A, 360, 499

Tautvaišiene, G., Edvardsson, B., Puzeras, E., \& Ilyin, I. 2005, A\&A, 431, 933

Tautvaišienè, G., Edvardsson, B., Puzeras, E., Barisevičius, G., \& Ilyin, I. 2010, MNRAS, 409, 1213

Tautvaišienè, G., Barisevičius, G., Chorniy, Y., Ilyin, I., \& Puzeras, E. 2013, MNRAS, 430, 621

Twarog, B. A., Ashman, K. M., \& Anthony-Twarog, B. J. 1997, AJ, 114, 2556

Ulrich, R. K. 1972, ApJ, 172, 165

Wachlin, F. C., Miller Bertolami, M. M., \& Althaus, L. G. 2011, A\&A, 533, A139

Yong, D., Carney, B. W., \& Friel, E. D. 2012, AJ, 144, 95

1 Institute of Theoretical Physics and Astronomy, Vilnius University, A. Gostauto 12, 01108 Vilnius, Lithuania e-mail: grazina.tautvaisiene@tfai.vu.1t

2 Laboratoire Lagrange (UMR7293), Université de Nice Sophia Antipolis, CNRS, Observatoire de la Côte d'Azur, CS 34229, 06304 Nice Cedex 4, France

3 INAF - Osservatorio Astrofisico di Arcetri, Largo E. Fermi, 5, 50125 Florence, Italy

4 INAF - Osservatorio Astronomico di Bologna, via Ranzani 1, 40127 Bologna, Italy

5 Department for Astrophysics, Nicolaus Copernicus Astronomical Center, ul. Rabiańska 8, 87-100 Toruń, Poland 
G. Tautvaišienė et al.: The Gaia-ESO Survey: CNO abundances in the open clusters Trumpler 20, NGC 4815, and NGC 6705

6 ESO, Alonso de Cordova 3107, 19001 Santiago de Chile, Chile

7 Department of Astronomy, Indiana University, Bloomington, IN 47405, USA

8 Institut d'Astrophysique et de Géophysique, Université de Liège, Allée du 6 Août, Bât. B5c, 4000 Liège, Belgium

9 ASI Science Data Center, via del Politecnico SNC, 00133 Roma, Italy

10 Spanish Virtual Observatory, Centro de Astrobiología (INTACSIC), PO Box 78, 28691 Villanueva de la Caada, Madrid, Spain

11 Institute of Astronomy, University of Cambridge, Madingley Road, Cambridge CB3 OHA, UK

12 Astrophysics Group, Research Institute for the Environment, Physical Sciences and Applied Mathematics, Keele University, Keele, Staffordshire ST5 5BG, UK
13 INAF-Padova Observatory, Vicolo dell'Osservatorio 5, 35122 Padova, Italy

14 Lund Observatory, Department of Astronomy and Theoretical Physics, Box 43, 22100 Lund, Sweden

15 INAF-Osservatorio Astronomico di Palermo, Piazza del Parlamento 1, 90134 Palermo, Italy

16 Instituto de Astrofísica de Andalucía-CSIC, Apdo. 3004, 18080 Granada, Spain

17 Astrophysics Research Institute, Liverpool John Moores University, 146 Brownlow Hill, Liverpool L3 5RF, UK

18 Centro de Astrofísica, Universidade do Porto, Rua das Estrelas, 4150-762 Porto, Portugal Departamento de Física e Astronomia, Faculdade de Ciências, Universidade do Porto, Rua do Campo Alegre, 4169-007 Porto, Portugal 